JOURNAL OF

SYMPLECTIC GEOMETRY

Volume 2, Number 2, 177-206, 2004

\title{
TOWARD A TOPOLOGICAL CHARACTERIZATION OF SYMPLECTIC MANIFOLDS
}

\author{
Robert E. GompF
}

\begin{abstract}
A fibration-like structure called a hyperpencil is defined on a smooth, closed $2 n$-manifold $X$, generalizing a linear system of curves on an algebraic variety. A deformation class of hyperpencils is shown to determine an isotopy class of symplectic structures on $X$. This provides an inverse to Donaldson's program for constructing linear systems on symplectic manifolds. In dimensions $\leq 6$, work of Donaldson and Auroux provides hyperpencils on any symplectic manifold, and the author conjectures that this extends to arbitrary dimensions. In dimensions where this holds, the set of deformation classes of hyperpencils canonically maps onto the set of isotopy classes of rational symplectic forms up to positive scale, topologically determining a dense subset of all symplectic forms up to an equivalence relation on hyperpencils. In particular, the existence of a hyperpencil topologically characterizes those manifolds in dimensions $\leq 6$ (and perhaps in general) that admit symplectic structures.
\end{abstract}

\section{Introduction}

Symplectic structures, which are closed, nondegenerate 2-forms $\omega$ on an even-dimensional manifold $X$, can be thought of as skew-symmetric analogs of constant curvature Riemannian metrics. The nondegeneracy condition (that each nonzero tangent vector pairs nontrivially with some vector) is the same in each case, and closure $(d \omega=0)$ corresponds to a constant curvature condition in that it is a differential equation guaranteeing that all such structures of a given dimension are locally identical. The study of constant curvature manifolds reduces, through covering space theory, to that of discrete isometry groups of Euclidean, hyperbolic and spherical space, so it 
is natural to ask whether symplectic structures also have some sort of topological characterization. Gromov $[\mathbf{G r}],[\mathbf{M c S}$, Theorem 7.34], showed that an open manifold $X$ admits a symplectic structure for each choice of almostcomplex structure (up to homotopy) and class $[\omega] \in H_{\mathrm{dR}}^{2}(X)$, reducing the existence question for symplectic structures on open manifolds to that of almost-complex structures (homotopy theory for the tangent bundle). However, the case of closed manifolds is much more difficult. For example, there exist homeomorphic pairs of smooth 4-manifolds with isomorphic tangent bundles, such that one admits symplectic structures and the other does not $[\mathbf{T a}]$. (See also $[\mathbf{G S}],[\mathbf{K}]$.) In the present paper, we propose a solution to this problem, by introducing a topological structure called a hyperpencil, which we show determines a symplectic structure (up to isotopy). In dimensions $\leq 6$, and conjecturally in general, a closed manifold admits a symplectic structure if and only if it admits a hyperpencil, and a dense subset of all symplectic structures (up to isotopy and scale) can be described as a quotient of the set of deformation classes of hyperpencils.

The prototype for hyperpencils comes from algebraic geometry (Example 2.6(a)). If $X \subset \mathbb{C P}^{N}$ is a smooth $n$-dimensional algebraic variety, we obtain a linear $k$-system $f: X-B \rightarrow \mathbb{C P}^{k}$ on $X$ by intersecting $X$ with a transverse linear subspace $A$ of codimension $k+1$, setting $B=X \cap A$, and defining $f$ to be the restriction of a projectivized linear surjection $\mathbb{C P}^{N}-A \rightarrow \mathbb{C P}^{k}$. Thus, any algebraic variety inherits a canonical deformation class of linear $k$-systems from its embedding in $\mathbb{C P}^{N}$. One can use the resulting local structure on $X$ to formulate a definition of linear systems in the category of smooth manifolds. ([G3] studies the general case of this.) When $k$ equals 1 and $n$, respectively, generic prototypes yield Lefschetz pencils and (singular) branched coverings, both of which have been extensively studied by topologists. Lefschetz used Lefschetz pencils to study the topology of algebraic varieties (e.g., $[\mathbf{L}]$ ), and in recent decades these structures have also arisen in 4-manifold theory (e.g., [GS]). We wish to use linear systems to construct symplectic structures. To obtain the strongest theorem, we wish to use the weakest possible hypotheses. This suggests using the smallest possible value for $k$, since a linear $k$-system generates linear $\ell$-systems for $\ell \leq k$ (assuming the associated almost-complex structures behave reasonably as in the algebraic case) by composition with a generic projection $\mathbb{C P}^{k}-A^{\prime} \rightarrow \mathbb{C P}^{\ell}$. However, the fibers of a linear $k$-system have (real) dimension $2(n-k)$; when $n-k>1$ it is already difficult to know when the fibers admit symplectic structures. Thus, the optimal case seems to be when $k=n-1$, when generic fibers are oriented surfaces, so each has a unique symplectic form (i.e., area form in this dimension) up to isotopy and a constant scale factor. Hyperpencils (Definition 2.4) are a type of linear $(n-1)$-system derived from the algebraic prototype. We have aimed for the weakest possible hypotheses guaranteeing the existence of symplectic structures, allowing the ugliest 
possible local behavior. It seems likely that additional constraints should be added for other purposes; for example, it may be possible to deform any hyperpencil into a much nicer "generic" form.

Our Main Theorem 2.11 can be paraphrased as follows:

Theorem 1.1. Let $X$ be a smooth, closed, oriented manifold.

(a) For any hyperpencil on $X$, the space of suitably compatible almostcomplex structures $J$ is nonempty and contractible.

(b) Every such $J$ is tamed by a symplectic form on $X$ realizing a certain cohomology class determined by $f$, and the space of such forms is contractible (for $J$ fixed or suitably varying).

(c) There is a canonical map $\Omega: \mathcal{P}(X) \rightarrow \mathcal{S}(X)$, where $\mathcal{P}(X)$ is the set of deformation classes of hyperpencils on $X$, and $\mathcal{S}(X)$ is the set of all isotopy classes of symplectic forms on $X$.

We first consider (c). Deformations of hyperpencils are defined in Definition 2.7. Symplectic forms $\omega_{0}$ and $\omega_{1}$ are isotopic if there is a diffeomorphism $\varphi: X \rightarrow X$ isotopic to $\operatorname{id}_{X}$ with $\varphi^{*} \omega_{1}=\omega_{0}$. By Moser's Theorem [M], this is equivalent to the existence of a deformation (smooth family of symplectic forms $\left.\omega_{s}, 0 \leq s \leq 1\right)$ for which $\left[\omega_{s}\right] \in H_{\mathrm{dR}}^{2}(X)$ is constant. Theorem 2.11(c) characterizes the symplectic forms associated by $\Omega$ to a given hyperpencil, using the intermediate structure $J$ (see Definition 2.9 and Lemma 2.10). For example, it is easy to check that the standard Kähler form on an algebraic variety is associated in this manner to the deformation class of hyperpencils determined by its embedding in $\mathbb{C P}^{N}$. A more expository discussion of Theorem 2.11 (in a slightly earlier form) appears in [G2]. The original form of the theorem, that a 4-manifold with a Lefschetz pencil admits a symplectic structure, was first proved by the author in 1990, but remained unpublished (due to the emergence of a more direct way of constructing unusual symplectic 4-manifolds [G1]) until its expository appearance as [GS, Theorem 10.2.18 and Corollary 10.2.23].

The main motivation for Theorem 1.1 is the use of (c) in characterizing symplectic manifolds. The symplectic forms produced by the theorem are integral (i.e., with cohomology class in the image of $\left.H^{2}(X ; \mathbb{Z}) \rightarrow H_{\mathrm{dR}}^{2}(X)\right)$. Donaldson $[\mathbf{D}]$ has proven that any integral symplectic manifold (up to scale) admits an associated Lefschetz pencil, and Auroux $[\mathbf{A 1}]$ has obtained a similar result for linear 2 -systems. These results imply the $n=2,3$ cases, respectively, of the following conjecture (which is trivially true for $n \leq 1$ ):

Conjecture 1.2. Let $\omega$ be any integral symplectic form on a closed $2 n$ manifold $X$. Then for any sufficiently large integer $m$, the isotopy class of $m \omega$ lies in the image of $\Omega$.

The conjecture is still open for $n \geq 4$, motivating our attempt at the weakest possible definition of hyperpencils. However, Auroux has made some 
technical progress on the problem [A2]. One would ultimately expect hyperpencils arising from Donaldson-Auroux theory to have much nicer local properties than arbitrary hyperpencils, for example explicit holomorphic local models at the critical points. The conjecture leads to characterization of symplectic manifolds as follows. Up to scale, every rational cohomology class is integral, and the subspace $\mathcal{S}_{\mathbb{Q}}(X) \subset \mathcal{S}(X)$ of symplectic forms with $[\omega]$ rational is dense (since nondegeneracy is an open condition). Thus, we may define $\tilde{\Omega}: \mathcal{P}(X) \times \mathbb{Q}_{+} \rightarrow \mathcal{S}(X)$ so that $\tilde{\Omega}(\varphi, q)$ is obtained by rescaling $\Omega(\varphi)$ to make its cohomology class $q$ times a primitive integral class, and conclude:

Proposition 1.3. If Conjecture 1.2 holds for $X$, then the image of the canonical map $\tilde{\Omega}: \mathcal{P}(X) \times \mathbb{Q}_{+} \rightarrow \mathcal{S}(X)$ is the dense subset $\mathcal{S}_{\mathbb{Q}}(X) \subset \mathcal{S}(X)$.

Corollary 1.4. In dimensions where Conjecture 1.2 holds (e.g., dimensions $\leq 6)$, a closed manifold admits a symplectic structure if and only if it admits a hyperpencil. A closed 4-manifold admits a symplectic structure if and only if it admits a Lefschetz pencil with $B \neq \emptyset$.

The last statement of the corollary follows from Theorem 1.1(c) and [D], if we restrict to Lefschetz pencils such that each irreducible component of each singular fiber intersects $B$ (since these are hyperpencils); the stated version is proved directly in [GS, Theorem 10.2.28]. (There, the condition $B \neq \emptyset$ is contained in the definition of Lefschetz pencils.) In dimensions where Conjecture 1.2 holds, we have now topologically characterized manifolds admitting symplectic structures. From there, to topologically determine the dense subset $\mathcal{S}_{\mathbb{Q}}(X) \subset \mathcal{S}(X)$, it suffices to understand the following:

Conjecture 1.5. The fibers of $\tilde{\Omega}$ (or equivalently, of the map $\bar{\Omega}: \mathcal{P}(X) \rightarrow$ $\mathcal{S}_{\mathbb{Q}}(X) / \mathbb{Q}_{+}$determined by $\Omega$ ) are specified by a topologically defined equivalence relation on $\mathcal{P}(X)$.

This may be easier to prove for a stronger definition of hyperpencils. The main evidence for Conjecture 1.5 is that the theorems of Donaldson and Auroux come with uniqueness statements up to a notion of stabilization, which multiplies the cohomology classes by large integers. While this stabilization comes from analytical considerations on special families of linear systems, one might hope to topologically define stabilization maps in general, $\sigma_{k}: \mathcal{P}(X) \rightarrow \mathcal{P}(X), k \in \mathbb{Z}_{+}$, with $\sigma_{1}=\operatorname{id}_{\mathcal{P}(X)}, \sigma_{k} \circ \sigma_{\ell}=\sigma_{k \ell}$ and $\Omega \circ \sigma_{k}=k \Omega$, and realize the equivalence relation in Conjecture 1.5 by the definition $\varphi \sim \psi$ if and only if $\sigma_{k}(\varphi)=\sigma_{\ell}(\psi)$ for some $k, \ell \in \mathbb{Z}_{+}$. However, these stabilizations already seem complicated in dimension 4 . (For $\sigma_{2}$, see $[\mathbf{A K}]$.)

Our main tool for constructing symplectic structures is a method originally used by Thurston $[\mathbf{T}]$ in the context of surface bundles, to use a symplectic structure on the base to construct one on the total space. This 
method has been generalized to bundles with higher dimensional fibers (e.g., [McS, Theorem 6.3]) and to bundles with complex quadratic singularities [GS, Theorem 10.2.18], but we show (Theorem 3.1) that the method works for maps that may be very different from bundle projections. For example, it suffices to have a map that is $J$-holomorphic for suitable almost-complex structures, with suitable data in a neighborhood of each point preimage. (An almost-complex structure on a manifold $X$ is a complex structure on its tangent bundle $T X$, or equivalently a bundle map $J: T X \rightarrow T X$ covering $\operatorname{id}_{X}$ with $J \circ J=-\operatorname{id}_{T X}$, which we should interpret as multiplication by $i$. A map is then J-holomorphic if its derivative is complex linear. Complex structures on other vector bundles may be interpreted similarly.) We apply this method to a hyperpencil $f: X-B \rightarrow \mathbb{C P}^{n-1}$, starting from the standard symplectic form $\omega_{\text {std }}$ on $\mathbb{C P}^{n-1}$. To do this, we need a suitable almost-complex structure on $X$, and to prove uniqueness up to isotopy we must be able to find a 1-parameter family $J_{s}$ connecting any two such almost-complex structures. Thus, we need various lemmas for splicing together locally defined almost-complex structures. These are compiled into Lemma 3.2, whose proof comprises most of Section 4. To emphasize that the choice of almost-complex structure does not crucially affect the resulting symplectic forms, we define hyperpencils using only locally defined almost-complex structures and prove that the relevant space of global almost-complex structures is nonempty and contractible (Theorem 1.1(a)). For convenience, and to emphasize the topological nature of the hypotheses, we always work with $C^{0}$ almost-complex structures. Thus, our spaces of almost-complex structures will always be given the $C^{0}$-topology (or for noncompact $X$, its natural generalization, the compact-open topology). In contrast, we have much more flexibility in topologizing spaces of symplectic forms. For example, contractibility in Theorem 1.1(b) holds for all $C^{k_{-}}$ spaces of forms and Sobolev spaces in between. (See Theorem 2.11(b).) Our method for constructing symplectic structures has other applications besides Theorem 1.1. We study high-dimensional Lefschetz pencils and other linear systems in [G3], and locally holomorphic maps with 2-dimensional fibers in [G4].

Throughout the paper, orientations are crucial. If $V$ is a $2 n$-dimensional real vector space, any nondegenerate, skew-symmetric, bilinear form $\omega$ on $V$ induces an orientation, since its top exterior power is a volume form. A (linear) complex structure $J$ on $V$ induces an orientation obtained, as usual, from any complex isomorphism $(V, J) \cong \mathbb{C}^{n}=\mathbb{C} \times \cdots \times \mathbb{C}$ in the product orientation (where $(1, i)$ is a positively oriented real basis for $\mathbb{C}$ ). If $V$ is given to be an oriented vector space, we only consider (unless otherwise specified) complex structures and nondegenerate 2 -forms inducing the given orientation on $V$. For example, almost-complex structures and symplectic forms on oriented manifolds implicitly induce the given orientation. We 
let $\omega_{\text {std }}$ denote the standard symplectic form on $\mathbb{C P}^{k}$, normalized so that $\int_{\mathbb{C P}^{1}} \omega_{\text {std }}=1$, so $\left[\omega_{\text {std }}\right] \in H_{\mathrm{dR}}^{2}\left(\mathbb{C P}^{k}\right)$ is the hyperplane class $h$, Poincaré dual to $\left[\mathbb{C P}^{k-1}\right] \in H_{2 k-2}\left(\mathbb{C P}^{k}\right)$.

The author wishes to acknowledge Denis Auroux for helpful discussions.

\section{The main theorem}

To define hyperpencils, we need some preliminary definitions. We begin by generalizing some standard terminology for relating symplectic and complex structures.

Definition 2.1. Let $T: V \rightarrow W$ be a linear transformation between finitedimensional real vector spaces, and let $\omega$ be a skew-symmetric bilinear form on $W$. A linear complex structure $J: V \rightarrow V$ will be called $(\omega, T)$-tame if $T^{*} \omega(v, J v)>0$ for all $v \in V-\operatorname{ker} T$. If, in addition, $T^{*} \omega$ is $J$-invariant (i.e., $T^{*} \omega(J v, J w)=T^{*} \omega(v, w)$ for all $\left.v, w \in V\right)$, we will call $J(\omega, T)$-compatible. For a $C^{1}$-map $f: X \rightarrow Y$ between manifolds, with a 2-form $\omega$ on $Y$, an almost-complex structure $J$ on $X$ will be called $(\omega, f)$-tame (resp. $(\omega, f)$ compatible) if it is $\left(\omega, d f_{x}\right)$-tame (resp. $\left(\omega, d f_{x}\right)$-compatible) for each $x \in X$. If $T=\operatorname{id}_{V}$ or $f=\operatorname{id}_{X}$, we will shorten the terminology to $\omega$-tame and $\omega$-compatible.

The last sentence of the definition is standard terminology. If $\omega$ tames some $J$ (i.e., $T=\operatorname{id}_{V}$ and $J$ is $\omega$-tame), then $\omega$ is obviously nondegenerate, so a closed, taming 2 -form $\omega$ is automatically symplectic. An $\omega$-tame $J$ induces the same orientation as $\omega$. (For example, homotope $\omega$ through taming forms to a compatible one.) A key advantage of $\omega$-taming over $\omega$ compatibility is that the former is an open condition on manifolds. In fact, the $\omega$-taming condition is satisfied provided that it holds on the unit sphere bundle in $T X$, so it is preserved under $\varepsilon$-small perturbations of $J$ and $\omega$ when $X$ is compact. If $J$ is $(\omega, T)$-tame then $\operatorname{ker} T$ is a $J$-complex subspace of $V$, so $\operatorname{Im} T \subset W$ inherits an $\omega$-tame complex structure $T_{*} J$ making $T$ complex linear $\left(T \circ J=T_{*} J \circ T\right)$. This will be $\omega$-compatible if and only if $J$ is $(\omega, T)$-compatible. For an $(\omega, f)$-tame almost-complex structure, preimages of regular values of $f$ will be $J$-holomorphic submanifolds (i.e., $J$ preserves their tangent spaces), and the complex structures induced on the fibers of $\operatorname{Im} d f \subset f^{*} T Y \rightarrow X$ will be denoted $f_{*} J$. Both the taming and compatibility conditions are preserved under taking convex combinations $\sum t_{i} \omega_{i}\left(\right.$ all $t_{i} \geq 0, \sum t_{i}=1$ ) for fixed $f, J$. An almost-complex symplectic manifold $(Y, J, \omega)$ is called almost-Kähler if $J$ is $\omega$-compatible, and Kähler if, in addition, $(Y, J)$ is a complex manifold. In either case, if $f: X \rightarrow Y$ is $J$-holomorphic for some almost-complex structure on $X$, this structure is $(\omega, f)$-compatible.

To prove uniqueness of symplectic forms induced by hyperpencils, we need a technical condition for critical points. Suppose $E, F \rightarrow X$ are real 
(finite dimensional) vector bundles over a metrizable topological space, and $T: E \rightarrow F$ is a (continuous) section of the bundle $\operatorname{Hom}(E, F)$. In our main application, these will be induced by a $C^{1}$-map $f: X \rightarrow Y$ between manifolds, with $T=d f: T X \rightarrow f^{*} T Y$. Motivated by this example, we call a point $x \in X$ regular if $T_{x}: E_{x} \rightarrow F_{x}$ is onto and critical otherwise. Let $P \subset E$ be the closure cl $\left(\bigcup \operatorname{ker} T_{x}\right)$, where $x$ varies over all the regular points of $T$ in $X$, and let $P_{x}=P \cap E_{x}$. Thus, $P_{x}=\operatorname{ker} T_{x}$ if $x$ is regular, and otherwise $P_{x} \subset \operatorname{ker} T_{x}$ consists of limits of sequences of vectors annihilated by $T$ at regular points.

Definition 2.2. A point $x \in X$ is wrapped if span $P_{x}$ has (real) codimension at most 2 in $\operatorname{ker} T_{x}$.

Proposition 2.3. Suppose that in a neighborhood of a critical point $x \in X$, $T$ is given by $d f$, for some holomorphic map $f: U \rightarrow \mathbb{C}^{n-1}$ with $U$ open in $\mathbb{C}^{n}$. If each fiber $f^{-1}(y)$ intersects the critical set $K$ of $f$ in at most a finite set, then $x$ is wrapped. In fact, $P_{x}=\operatorname{ker} T_{x}$.

This proposition will show that our hypothesis of wrapped critical points is broad enough to be useful. Note, however, that the proposition becomes false without the finiteness hypothesis, e.g., $n=3, f(x, y, z)=\left(x^{2}, y^{2}\right)$ at $(0,0,0)$. (For $n=2, P_{x}$ equals $\operatorname{ker} T_{x}$ unless $f$ is constant or $x$ is a smooth point of $f$ with multiplicity $>1$; cf. [G4, proof of Proposition 1.3].) Similarly, $P_{x}$ may not equal $\operatorname{ker} T_{x}$ if we pass from the holomorphic setting to $C^{\infty}$. (The $C^{\infty}$-map $f: \mathbb{R}^{2} \rightarrow \mathbb{R}$ given by $f(x, y)=y^{3}+e^{-1 / x^{2}} y$ has a unique critical point at $(0,0)$, so $d f_{(0,0)}=0$, but $P_{(0,0)}$ is the $x$-axis.)

Proof. For $\ell \geq 2$, let $K_{\ell} \subset K$ be the set of $z \in U$ for which ker $d f_{z}$ has complex dimension $\geq \ell$. Thus, $K=K_{2} \supset K_{3} \supset \cdots \supset K_{n+1}=\emptyset$. We begin by showing that each $K_{\ell}$ is an analytic variety of complex codimension $\geq \ell$ in $U$. Analyticity follows immediately from the description of $K_{\ell}$ as the set of $z \in U$ for which every $(n-\ell+1) \times(n-\ell+1)$ submatrix of $d f_{z}$ has determinant zero. For $z$ in the top stratum $W$ of $K_{\ell}$, let $Q_{z}=$ $T_{z} W \cap \operatorname{ker} d f_{z}$. If $\operatorname{codim}_{\mathbb{C}} K_{\ell}<\ell$, then $Q_{z}$ has nonzero dimension for all $z \in W$. Choose some $z_{0} \in W$ minimizing this dimension. To see that $Q$ is a smooth distribution on $W$ near $z_{0}$, choose a projection $\pi$ of $\mathbb{C}^{n-1}$ whose restriction to $d f_{z_{0}}\left(T_{z_{0}} W\right)$ is an isomorphism, and note that $\pi \circ f \mid W$ is a submersion at $z_{0}$ with $\operatorname{ker} d(\pi \circ f \mid W)_{z}$ containing $\operatorname{ker} d(f \mid W)_{z}=Q_{z}$; these latter spaces are then equal near $z_{0}$ by minimality of $\operatorname{dim} Q_{z_{0}}$. Now choose a smooth, nonzero vector field in $Q$ near $z_{0}$. By integrating, we obtain a curve in $W \subset K$ whose image under $f$ is a point $y$, contradicting finiteness of $f^{-1}(y) \cap K$.

Now observe that the subset $V=\bigcup_{z \in U} \operatorname{ker} d f_{z} \subset T U=U \times \mathbb{C}^{n}$ is an analytic variety with complex dimension $\geq n+1$ everywhere, since it is cut out by the system of $n-1$ equations $d f_{z}(v)=0$ in $(z, v)$. For each $\ell \geq 2$, 
the subset $V_{\ell}=V \cap\left(\left(K_{\ell}-K_{\ell+1}\right) \times \mathbb{C}^{n}\right)$ is a complex $\ell$-plane bundle over $K_{\ell}-K_{\ell+1}$, and the latter has codimension $\geq \ell$ in $U$, so $\operatorname{dim}_{\mathbb{C}} V_{\ell} \leq n<$ $\operatorname{dim}_{\mathbb{C}} V$. Thus, $V-K \times \mathbb{C}^{n}=V-\bigcup_{\ell=2}^{n} V_{\ell}$ has closure $V$ in $U \times \mathbb{C}^{n}$. The proposition follows immediately.

Definition 2.4. A hyperpencil on a smooth, closed, oriented, $2 n$-manifold $X$ is a (necessarily finite) subset $B \subset X$ called the base locus and a smooth map $f: X-B \rightarrow \mathbb{C P}^{n-1}$ such that

(1) each $b \in B$ is mapped to $0 \in \mathbb{C}^{n}$ by an orientation-preserving local coordinate chart in which $f$ is given by projectivization $\mathbb{C}^{n}-\{0\} \rightarrow$ $\mathbb{C} \mathbb{P}^{n-1}$,

(2) each critical point of $f$ is wrapped and has a neighborhood with a continuous $\left(\omega_{\text {std }}, f\right)$-compatible almost-complex structure, and

(3) each fiber $F_{y}=\mathrm{cl} f^{-1}(y) \subset X$ contains only finitely many critical points of $f$, and each component of each $F_{y}-\{$ critical points $\}$ intersects $B$.

Remarks 2.5. (a) The results in this paper are all trivially true for $n \leq 1$ (after Moser $[\mathbf{M}]$ ), so we will assume $n \geq 2$ whenever convenient. For potential applications such as sub-hyperpencils, where it may be convenient to allow $n \leq 1$, we specify the required conventions: For $n=0, B$ equals $X$, and a symplectic form on a 0 -manifold is its unique positive orientation. For $n=1$, we require $B \subset X$ to be finite, and its Poincaré dual in $H_{\mathrm{dR}}^{2}(X)$ is the class $c_{f}$ used in Theorem 2.11.

(b) By Condition (1), the fibers $F_{y}$ of a hyperpencil are complex lines near each $b \in B$. Thus, $F_{y}=f^{-1}(y) \cup B$ and $F_{y} \cap F_{z}=B$ for $y \neq z$, so $B$ is closed and discrete, hence finite. Each $F_{y}$ is an oriented surface (in the preimage orientation) except possibly at finitely many singularities, where $F_{y}$ intersects the critical set of $f$ in $X-B$.

(c) For $n \leq 2$ the hypothesis of wrapped critical points is trivially true. (The proof of Main Theorem 2.11 shows that the regular points of $f$ are dense in $X-B$, so each $\operatorname{span}_{\mathbb{R}} P_{x} \subset T_{x}(X-B)$ is a nontrivial complex subspace.) For $n=3$, this hypothesis can be eliminated if we assume that at each point in the closure of the set of unwrapped critical points, the given local almostcomplex structure makes $f J$-holomorphic for some continuous, $\omega_{\text {std }}$-tame local complex structure on the bundle $f^{*} T \mathbb{C P}^{n-1}$. (In fact, an even weaker hypothesis guarantees the Main Theorem when $n=3$, namely $\left(\omega_{\text {std }}, d f\right)$ extendability as used in Addendum 3.3 with $C=D=\emptyset$ and $E=T X$.) For arbitrary $n$, the hypothesis of wrapped critical points can be dropped in the presence of a global $\omega_{\text {std }}$-compatible complex structure (standard near $B$ ) on $f^{*} T \mathbb{C P}^{n-1}$ making $f J$-holomorphic for each local almost-complex structure on $X-B$, but the resulting isotopy class of symplectic forms could then conceivably depend on the choice of this structure on $f^{*} T \mathbb{C P}^{n-1}$. 
(d) Throughout the article, we use continuous, rather than smooth, almostcomplex structures. This is both for convenience (avoiding awkward and unnecessary proofs of smoothness) and to emphasize that the purpose of the local almost-complex structures is topological rather than analytical, controlling monodromy around the critical values. For a Lefschetz pencil on a 4-manifold, for example, the monodromy consists of right-handed Dehn twists. Allowing the opposite handedness violates the hypothesis of (compatibly oriented) local almost-complex structures, and results in manifolds having no symplectic structure.

(e) It is an open question whether $\left(\omega_{\text {std }}, f\right)$-compatibility can be replaced by $\left(\omega_{\text {std }}, f\right)$-taming. This could be done throughout the paper if Question 4.3 had an affirmative answer, and can also be done in the situation at the end of (c) above (arbitrary $n$ ).

Examples 2.6. (a) Any smooth algebraic variety $X \subset \mathbb{C P}^{N}$ admits a hyperpencil. Simply pick a linear subspace $A \approx \mathbb{C P}^{N-n}\left(n=\operatorname{dim}_{\mathbb{C}} X\right)$ transverse to $X$ in $\mathbb{C P}^{N}$, let $B=X \cap A$ and let $f$ be the restriction of the holomorphic projection $\mathbb{C P}^{N}-A \rightarrow \mathbb{C P}^{n-1}$. Condition (1) follows from transversality. For Condition (3), note that each fiber $F_{y}$ is an algebraic curve in some linear subspace of $\mathbb{C P}^{N}$ containing $A$ as a codimension-1 subspace. Thus, each irreducible component of $F_{y}$ intersects $A$, and so has a 0 -dimensional intersection with the critical set (since Condition (1) locates some regular points). Now the critical points of $f$ are wrapped by Proposition 2.3, and the obvious holomorphic structure on $X$ completes Condition (2).

(b) On 4-manifolds, Lefschetz pencils are inspired by the above Example (a) with $n=2$. Their definition is obtained from Definition $2.4(n=2)$ by replacing Conditions (2) and (3) with the condition that $f$ should locally be a complex Morse function, i.e., modeled by $f\left(z_{1}, z_{2}\right)=z_{1}^{2}+z_{2}^{2}$ at each critical point. (This holds in (a) for a generic choice of $A$.) See [GS] for a recent survey on Lefschetz pencils. Note that Condition (2) is automatically satisfied by Lefschetz pencils, as is the finiteness part of Condition (3). Lefschetz pencils need not satisfy the other part of Condition (3), however. This condition on $F_{y} \cap B$, which guarantees that a (4-dimensional) Lefschetz pencil is a hyperpencil, is actually not necessary for constructing symplectic structures on Lefschetz pencils. (See [GS, Theorem 10.2.18 and Corollary 10.2.23] for details.) It suffices to know either that $B \neq \emptyset$ or that the fibers are nontrivial in $H_{2}(X ; \mathbb{R})$. (In fact, the only counterexamples are torus bundles, namely $L(p, 1) \times S^{1} \rightarrow S^{2}$, and their blowups; see [GS, Remark 10.2.22(a)].) However, without Condition (3), one loses control of the cohomology class of the resulting form, and with it one loses uniqueness of the isotopy class. 
To define an appropriate equivalence relation among hyperpencils on $X$, we again work by analogy with algebraic geometry. We begin by organizing hyperpencils into families over a parameter space $S$. Roughly, these families are given by bundles over $S$ whose fibers have continuously varying hyperpencil structures.

Definition 2.7. A family of hyperpencils parametrized by a topological space $S$ consists of a pair of fiber bundles $\pi_{X}: X \rightarrow S, \pi_{Y}: Y \rightarrow S$, a subset $B \subset X$ and a continuous, fiberwise smooth map $f: X-B \rightarrow Y$ covering $\operatorname{id}_{S}$, subject to the following: The fibers $X_{s}$ of $X, s \in S$, are all diffeomorphic to a fixed, closed, oriented $2 n$-manifold (so the structure group consists of orientation-preserving diffeomorphisms of the fiber in the $C^{\infty}$-topology), and the fibers of $Y$ are diffeomorphic to $\mathbb{C P}^{n-1}$ with structure group $\mathbb{P U}(n)$ acting in the usual way. The map $\pi_{X} \mid B: B \rightarrow S$ is a (necessarily finite) covering map. In addition:

(1) $B$ has a neighborhood $V \subset X$ on which $\pi_{X} \mid V: V \rightarrow S$ lifts to $\tilde{\pi}$ : $V \rightarrow B$, and $\tilde{\pi}$ is given the structure of a $\mathrm{U}(n)$-vector bundle (with zero section $B$ and fibers oriented compatibly with those of $\pi_{X}$ ). The map $f \mid V-B$ is projectivization on each fiber of $\tilde{\pi}$.

(2) The map $d f: T^{v}(X-B) \rightarrow f^{*} T^{v} Y$ (where $T^{v}$ denotes the bundle of tangent spaces to the fibers of $\pi_{X}$ and $\pi_{Y}$ ) is continuous, and each critical point of $d f$ has a neighborhood in $X-B$ over which $T^{v} X$ has an $\left(\omega_{\text {std }}, d f\right)$-compatible complex vector bundle structure.

(3) For each fiber $X_{s}$ of $\pi_{X}$, each critical point of $f \mid X_{s}-B$ is wrapped (in $X_{s}$ ), and Condition (3) of Definition 2.4 is satisfied.

A deformation of hyperpencils is a family parametrized by $I=[0,1]$ with $X=I \times X_{0}$.

It is easily verified that a family of hyperpencils parametrized by a 1-point space is the same as a hyperpencil (together with a fixed choice of the charts in Definition 2.4(1) up to $\mathrm{U}(n)$ action). If $\varphi: S \rightarrow S^{\prime}$ is continuous, then a family of hyperpencils parametrized by $S^{\prime}$ pulls back to one parametrized by $S$. For example, any parametrized family of hyperpencils restricts to a hyperpencil on each $X_{s}$, or to a family parametrized by any subspace of $S$. Now, we easily obtain an equivalence relation by calling two hyperpencils on a fixed manifold deformation equivalent if they are realized as $X_{0}$ and $X_{1}$ for some deformation. (The only technicality is that in transitivity, the middle hyperpencil may inherit two different sets of the charts in Definition 2.4(1). However, these charts can easily be changed, by triviality of bundles over $I$. Closer inspection also shows that we can find a continuous family interpolating between any two such charts; cf. proof of Lemma 2.10.) For a parametrized family with $S$ path connected, any $X_{s}$ and $X_{t}$ can be identified so that their hyperpencils are deformation equivalent. We can 
also assume the deformation is constant near each endpoint of $I$. If, in addition, $X=S \times X_{s}$ is the trivial bundle, we obtain a family of deformation equivalent hyperpencils on the fixed manifold $X_{s}$.

Examples 2.8. (a) To construct families of hyperpencils as in Example 2.6(a), let $G$ denote the complex Grassmann manifold of codimension- $n$ linear subspaces of $\mathbb{C P}^{N}$, and let $\gamma \subset G \times \mathbb{C P}^{N}$ be the tautological bundle whose fiber over $A \in G$ is $A \subset \mathbb{C P}^{N}$. If $Y \rightarrow G$ is the bundle whose fiber over $A$ is the $(n-1)$-plane in $\mathbb{C P}^{N}$ with maximal distance from $A$, we obtain a canonical holomorphic map $f: G \times \mathbb{C P}^{N}-\gamma \rightarrow Y$ covering $\operatorname{id}_{G}$, induced by linear projections on $\mathbb{C}^{N+1}$. Given an $n$-dimensional smooth algebraic variety $X_{0}$ in $\mathbb{C P}^{N}$, let $S \subset G$ be the open set of subspaces transverse to $X$. Restricting $f$ to a map $S \times X_{0}-\gamma \rightarrow Y \mid S$, we obtain a parametrized family of hyperpencils, consisting of all hyperpencils on $X_{0}$ obtained by Example 2.6(a). (The given holomorphic structure on $S \times X_{0}$ satisfies Condition (2) above.) For example, when $n=2$, generic members of the family will be Lefschetz pencils, but there will typically also be parameter values for which quadratic critical points coalesce into those of higher degree. In general, the space $S$ is path connected (since it is obtained from $G$ by removing a subvariety of positive complex codimension), so we conclude that all hyperpencils on $X_{0}$ obtained by Example 2.6(a) are deformation equivalent (for a fixed embedding $X_{0} \subset \mathbb{C P}^{N}$ ).

(b) For a family in which $X$ is a nontrivial bundle, note that the space of all hypersurfaces of a fixed degree in $\mathbb{C P}^{N}$ is parametrized by some $\mathbb{C} \mathbb{P}^{M}$. Let $S \subset G \times \mathbb{C P}^{M}$ denote the path-connected subset of pairs $(A, t)$ such that the variety $X_{t}$ is nonsingular and transverse to $A$. The construction of (a) above generalizes immediately to produce a parametrized family consisting of all hyperpencils as above on all nonsingular hypersurfaces of a fixed degree. It follows that any two nonsingular hypersurfaces of the same degree in $\mathbb{C P}^{N}$ are diffeomorphic in such a way that the canonical families of hyperpencils are deformation equivalent. We also see that the canonical deformation class of hyperpencils on a fixed hypersurface is invariant under self-diffeomorphisms induced by monodromy of the bundle of all nonsingular hypersurfaces.

We relate hyperpencils $f: X-B \rightarrow \mathbb{C P}^{n-1}$ to symplectic structures $\omega$ on $X$ via the existence of local almost-complex structures that are simultaneously $\omega$-tame and $\left(\omega_{\text {std }}, f\right)$-compatible:

Definition 2.9. Let $\omega$ be a continuous 2-form on an oriented manifold $X$. A hyperpencil $f: X-B \rightarrow \mathbb{C P}^{n-1}$ is $\omega$-tame if $X$ is covered by open sets $W_{\alpha}$ with continuous, $\omega$-tame almost-complex structures $J_{\alpha}$ such that $J_{\alpha} \mid W_{\alpha}-B$ is $\left(\omega_{\text {std }}, f\right)$-compatible, and the structures $f_{*} J_{\alpha}$ on $\operatorname{Im} d f \subset$ $f^{*} T \mathbb{C P}^{n-1} \mid W_{\alpha}-B$ all agree where their domains overlap. Similarly, a family 
$f: X-B \rightarrow Y$ of hyperpencils parametrized by $S$ is $\omega$-tame for a continuous family $\omega$ of 2 -forms on the fibers of $\pi_{X}$, if $X$ is covered by $\left(W_{\alpha}, J_{\alpha}\right)$ as above. (Here $W_{\alpha}$ is open in $X, J_{\alpha}$ is a complex vector bundle structure on $T^{v} X \mid W_{\alpha}$, and the structures $f_{*} J_{\alpha}$ fit together on $\operatorname{Im} d f \subset f^{*} T^{v} Y$.)

Lemma 2.10. A hyperpencil $f$ is $\omega$-tame if and only if there is a global, continuous, $\omega$-tame almost-complex structure $J$ on $X$ with $J \mid X-B\left(\omega_{\text {std }}, f\right)$ compatible. The structure $J$ can be chosen to agree near $B$ with the local complex structures induced by any preassigned charts as in Definition 2.4(1), and so that $f_{*} J$ agrees with the structures $f_{*} J_{\alpha}$ given by Definition 2.9 if these are standard near $B$. The corresponding statements also hold for a family of hyperpencils parametrized by a metrizable space, where $J$ is a complex vector bundle structure on $T^{v} X$ agreeing on a neighborhood of $B$ with the fiberwise complex structure determined by the vector bundle in Definition 2.7(1).

In other words, the local structures $J_{\alpha}$ of Definition 2.9 can be patched together to form a global structure. The "if" direction of this lemma is obvious; the other is proved in Section 4.

Any hyperpencil $f: X-B \rightarrow \mathbb{C P}^{n-1}$ determines a class $c_{f}=f^{*} h \in$ $H^{2}(X ; \mathbb{Z}) \cong H^{2}(X-B ; \mathbb{Z})$, where $h$ is the hyperplane class, Poincaré dual to $\left[\mathbb{C P}^{n-2}\right] \in H_{2 n-4}\left(\mathbb{C P}^{n-1} ; \mathbb{Z}\right)$. This class in $X$ is invariant under deformations of $f$. When $X \subset \mathbb{C P}^{N}$ is a smooth algebraic variety with its canonical deformation class of hyperpencils, $c_{f}$ is the restriction of the hyperplane class of $\mathbb{C P}^{N}$, and in general for $n=2, c_{f}$ is Poincaré dual to any fiber $F_{y}$. We also use $c_{f}$ to denote the corresponding class in $H_{\mathrm{dR}}^{2}(X)$; this is the cohomology class of the symplectic forms associated to $f$.

We are now ready to state the Main Theorem.

Theorem 2.11. Let $X$ be a smooth, closed, oriented $2 n$-manifold.

a) For any hyperpencil $f: X-B \rightarrow \mathbb{C P}^{n-1}$ there is a continuous almostcomplex structure $J$ on $X$ that is $\left(\omega_{\text {std }}, f\right)$-compatible on $X-B$, and agrees near $B$ with the complex structure given there by Definition 2.4(1) (for any fixed choice of charts). The space of all such structures $J$ in the $C^{0}$-topology (for fixed $f$ and charts near $B$ ) is contractible, as is the space of all $J$ on $X$ that are $\left(\omega_{\text {std }}, f\right)$-compatible on $X-B$ (without the constraint near $B$ ).

b) For any $J$ as in the first sentence of (a), there is a symplectic structure $\omega$ on $X$ with $[\omega]=c_{f} \in H_{\mathrm{dR}}^{2}(X)$, taming $J$ (so that $f$ is $\omega$-tame as in Definition 2.9). For fixed $J$, such structures $\omega$ form a convex subset of the space of all closed 2-forms. If only $f$ and $f_{*} J$ are fixed (otherwise allowing $J$ to vary), the space of such (smooth) symplectic structures is still contractible in any $C^{k}$-topology, $0 \leq k \leq \infty$, or locally convex metric topology in between, as is the completion in such a metric. 
c) For each deformation class $\varphi$ of hyperpencils on $X$, there is a unique isotopy class of symplectic forms on $X$ containing representatives $\omega$ for which some $f \in \varphi$ is $\omega$-tame and $[\omega]=c_{f}$.

There are also versions of (a) and (b) for parametrized families of hyperpencils; see Lemmas 3.2 (as applied in proving Theorem 2.11(a)) and 3.4, respectively. The completion in (b) means to complete the space of 2 -forms, take the closure of the affine subspace with $[\omega]=c_{f}$, and restrict to an open subset using the taming condition. Examples include the $C^{k}$-spaces of taming forms, $0 \leq k \leq \infty$, and many Sobolev spaces. The result could be stated in even further generality; see Theorem 16 of $[\mathbf{P}]$.

Example 2.12. For a smooth algebraic variety $X \subset \mathbb{C P}^{N}$, the standard holomorphic structure on $X$ is obviously $\left(\omega_{\text {std }} \mid X\right)$-tame. Thus the isotopy class of symplectic structures determined by the canonical deformation class of hyperpencils (Example 2.6(a)) is the one containing the standard Kähler form $\omega_{\text {std }} \mid X$.

\section{Proof of the Main Theorem 2.11}

Our main tool for constructing symplectic structures is the following theorem. This is based on an idea, which was used by Thurston $[\mathbf{T}]$ to construct symplectic structures on total spaces of surface bundles over symplectic manifolds, and which generalizes to bundles with symplectic fibers of arbitrary dimension (e.g., [McS, Theorem 6.3]). We now generalize still further, from bundle projections to singular maps suitably controlled by almost-complex structures, and work relative to a subset $C$ of the domain. The result is general enough to apply to hyperpencils, and also has other applications [G3], [G4].

Theorem 3.1. Let $f: X \rightarrow Y$ be a smooth map between manifolds, with $X$ - int $C$ compact for some closed subset $C$ with a neighborhood $W_{C}$ in $X$. Suppose that $\omega_{Y}$ is a symplectic form on $Y$, and $J$ is a continuous, $\left(\omega_{Y}, f\right)$ tame almost-complex structure on $X$. Fix a class $c \in H_{\mathrm{dR}}^{2}(X)$. Suppose that for each $y \in Y, f^{-1}(y)$ has a neighborhood $W_{y}$ in $X$ containing $W_{C}$, with the restriction $H_{\mathrm{dR}}^{1}\left(W_{y}\right) \rightarrow H_{\mathrm{dR}}^{1}\left(W_{C}\right)$ surjective, and with a closed 2-form $\eta_{y}$ on $W_{y}$ such that $\left[\eta_{y}\right]=c \mid W_{y} \in H_{\mathrm{dR}}^{2}\left(W_{y}\right)$ and such that $\eta_{y}$ tames $J$ on each of the complex subspaces ker $d f_{x}, x \in W_{y}$. Suppose that these forms $\eta_{y}$ all agree on $W_{C}$, and that the resulting form $\eta_{C}$ on $W_{C}$ tames $J$ on $T X \mid C$. Then there is a closed 2-form $\eta$ on $X$ agreeing with $\eta_{C}$ near $C$ with $[\eta]=c \in H_{\mathrm{dR}}^{2}(X)$, and such that for all sufficiently small $t>0$ the form $\omega_{t}=t \eta+f^{*} \omega_{Y}$ on $X$ tames $J$ (and hence is symplectic).

To show why this theorem generalizes Thurston's construction, we obtain the latter as a special case. (See $[\mathbf{G 2}]$ for additional details.) Suppose $f$ : 
$X \rightarrow Y$ is a fiber bundle with $X$ compact. Then we can take $C$ and $W_{C}$ to be empty. The required hypotheses for Thurston's construction (e.g., as given in $[\mathbf{M c S}])$ include symplectic structures on $Y$ and the fibers $f^{-1}(y)$, which give almost-complex structures on $Y$ and the subbundle of $T X$ tangent to the fibers. These can easily be combined into an almost-complex structure $J$ on $X$ that makes $f J$-holomorphic and hence is $\left(\omega_{Y}, f\right)$-tame. The final hypothesis for Thurston's construction guarantees the existence of a suitable class $c$, and the remaining hypotheses of Theorem 3.1 are now easily verified (cf. [G2]). The family of symplectic forms resulting from Theorem 3.1 is the same one obtained by Thurston.

The proof of Theorem 3.1 is obtained by modifying Thurston's method to exploit the almost-complex structure, allowing us to deal with a complicated critical set for $f$ and work relative to a possibly nonempty subset $C$.

Proof. Fix a representative $\zeta$ of the deRham class $c$. For each $y \in Y$, $\left[\eta_{y}\right]=c \mid W_{y}$, so we can write $\eta_{y}=\zeta+d \alpha_{y}$ for some 1 -form $\alpha_{y}$ on $W_{y}$. Pick some $y_{0} \in Y$ and set $\alpha_{C}=\alpha_{y_{0}} \mid W_{C}$. Then for each $y, d\left(\alpha_{C}-\alpha_{y}\right)=$ $\left(\eta_{y_{0}}-\zeta\right)-\left(\eta_{y}-\zeta\right)=0$ on $W_{C}$, so $\left[\alpha_{C}-\alpha_{y}\right] \in H_{\mathrm{dR}}^{1}\left(W_{C}\right)$ is defined. By hypothesis, any such class extends to $H_{\mathrm{dR}}^{1}\left(W_{y}\right)$, so after adding a closed form on $W_{y}$ to $\alpha_{y}$, we can assume $\alpha_{C}-\alpha_{y}$ is exact on $W_{C}$. Choosing a function $g: W_{y} \rightarrow \mathbb{R}$ with $d g=\alpha_{C}-\alpha_{y}$ near $C$, and replacing $\alpha_{y}$ by $\alpha_{y}+d g$, we obtain that $\alpha_{y}=\alpha_{C}$ near $C$ for each $y$. Since each $X-W_{y}$ is compact, each $y \in Y$ has a neighborhood disjoint from $f\left(X-W_{y}\right)$. Thus, we can cover $Y$ by open sets $U_{i}$, with each $f^{-1}\left(U_{i}\right)$ contained in some $W_{y}$. Let $\left\{\rho_{i}\right\}$ be a subordinate partition of unity on $Y$. The corresponding partition of unity $\left\{\rho_{i} \circ f\right\}$ on $X$ can be used to splice the forms $\alpha_{y}$; let $\eta=\zeta+d \sum_{i}\left(\rho_{i} \circ f\right) \alpha_{y_{i}}$. Clearly, $\eta$ is closed with $[\eta]=[\zeta]=c \in H_{\mathrm{dR}}^{2}(X)$, and $\eta=\eta_{C}$ near $C$, so it suffices to show that $\omega_{t}$ tames $J(t>0$ small). In preparation, perform the differentiation to obtain $\eta=\zeta+\sum_{i}\left(\rho_{i} \circ f\right) d \alpha_{y_{i}}+\sum_{i}\left(d \rho_{i} \circ d f\right) \wedge \alpha_{y_{i}}$. The last term vanishes when applied to a pair of vectors in ker $d f_{x}$, so on each ker $d f_{x}$ we have $\eta=\zeta+\sum_{i}\left(\rho_{i} \circ f\right) d \alpha_{y_{i}}=\sum_{i}\left(\rho_{i} \circ f\right) \eta_{y_{i}}$. By hypothesis, this is a convex combination of taming forms, so we conclude that $J \mid \operatorname{ker} d f_{x}$ is $\eta$-tame for each $x \in X$.

It remains to show that there is a $t_{0}>0$ for which $\omega_{t}(v, J v)>0$ for every $t \in\left(0, t_{0}\right)$ and $v$ in the unit sphere bundle $\Sigma \subset T X$ (for any convenient metric). But

$$
\omega_{t}(v, J v)=t \eta(v, J v)+f^{*} \omega_{Y}(v, J v) .
$$

Since $J$ is $\left(\omega_{Y}, f\right)$-tame, the last term is positive for $v \notin$ ker $d f$ and zero otherwise. Since $J \mid$ ker $d f$ is $\eta$-tame, the continuous function $\eta(v, J v)$ is positive for all $v$ in some neighborhood $U$ of $\operatorname{ker} d f \cap \Sigma$ in $\Sigma$. Similarly, for $v \in \Sigma \mid C, \eta(v, J v)=\eta_{C}(v, J v)>0$. Thus, $\omega_{t}(v, J v)>0$ for all $t>0$ when $v \in U \cup \Sigma \mid C$. On the compact set $\Sigma \mid(X-\operatorname{int} C)-U$ containing the rest of $\Sigma, \eta(v, J v)$ is bounded and the last displayed term is bounded below by 
a positive constant, so $\omega_{t}(v, J v)>0$ for $0<t<t_{0}$ sufficiently small, as required.

We also need some techniques for splicing together locally defined almostcomplex structures. These are given by the following lemma, whose proof appears in Section 4. As in Definition 2.2 (of wrapped critical points), we let $E, F \rightarrow X$ be real vector bundles over a metrizable space, with fiber dimensions $2 n$ and $2 n-2$ respectively, and this time equipped with fiber orientations. We again fix a section $T: E \rightarrow F$ of $\operatorname{Hom}(E, F)$. In the applications, $T$ will be $d f: T X \rightarrow f^{*} T Y$ for some $C^{1}$ map $f: X \rightarrow Y$, or a family of such maps, although the extra generality is useful in proving the lemma below. We wish to splice together locally defined complex structures on the bundle $E$, so as to extend a preassigned structure from some closed (possibly empty) subset $C \subset X$ to all of $X$. Our approach requires a restriction on either the topology of the critical points or the induced complex structures on the image $T(E) \subset F$. We assume the latter restriction on a closed subset $D$ (which without loss of generality contains $C$ ) and the former restriction elsewhere. The cases we require are when $D$ equals $C$ or $X$ (allowing us to set $V$ equal to $U$ or $X$ below), but the general case poses no additional difficulties. A 2-form on $E$ or $F$ will mean a continuously varying choice of a skew-symmetric bilinear form on each fiber of the bundle.

Lemma 3.2. For $E, F \rightarrow X$ and $T$ as above, let $C \subset D \subset X$ be closed subsets such that the regular points of $T \mid X-C$ are dense in $X-C$, and let $\omega_{F}$ be a nondegenerate 2-form on $F$ (inducing the given fiber orientation). For some neighborhood $U$ of $C$, let $J_{C}$ be an $\left(\omega_{F}, T\right)$-compatible complex structure on the (oriented) bundle $E \mid U$. Suppose that each $x \in X-U$ has a neighborhood $W_{x}$ with an $\left(\omega_{F}, T\right)$-compatible complex structure on $E \mid W_{x}$, and that for some neighborhood $V$ of $D$, these can be chosen for all $x \in V-U$ so that the induced structures on $T\left(E \mid W_{x}\right) \subset F \mid W_{x}$ agree with each other and with $T_{*} J_{C}$ wherever the domains overlap. Let $J_{D}$ denote the resulting complex structure on the fibers of $T(E \mid V)$.

a) If $n \geq 3$, assume each critical point of $T$ in $X-D$ is wrapped. Then $J_{C} \mid C$ extends to an $\left(\omega_{F}, T\right)$-compatible complex structure $J$ on $E$ with $T_{*} J \mid D=J_{D}$.

b) Suppose $D=X$ and $\omega_{E}$ is a 2-form on $E$. If the local complex structures on $E$ given above (including $J_{C}$ ) can be chosen to be $\omega_{E}$-tame, then we can assume $J$ is $\omega_{E}$-tame.

c) Suppose that $\partial C \subset X_{0}=X-\operatorname{int} C$ and (if $D \neq C$ ) $\partial D \subset X-\operatorname{int} D$ have disjoint collar neighborhoods compatible with the given structures on $E$ and F. (See below.) Then in both cases (a) and (b) above, the space $\mathcal{J}$ of all complex structures $J$ satisfying the given conclusions is weakly contractible when $X_{0}$ is locally compact and contractible when $X_{0}$ is compact. 
All of the above remains true if compatibility is replaced by taming everywhere, provided that $D=X$.

Recall that $\mathcal{J}$ is given the compact-open topology, which equals the $C^{0}$ topology when $X_{0}$ is compact. See the proof for further details. The "compatible collar" hypothesis on $\partial C$ means that there is a subset $K \subset X_{0}$ homeomorphic to $I \times \partial C$ with $\{0\} \times \partial C$ mapping to $\partial C$ in the obvious way and $\{1\} \times \partial C$ mapping onto $\partial K$ (the boundary in $X_{0}$ in the sense of general topology), and that $E \mid K$ and $F \mid K$ can be identified with $I \times(E \mid \partial C)$ and $I \times(F \mid \partial C)$ in such a way that $T, \omega_{F}, \omega_{E}$ (in case $\left.(\mathrm{b})\right)$, and $J_{D}($ if $D \neq C$ ) are constant over each $I \times\{x\} \subset I \times \partial C$.

The hypothesis of wrapped critical points can be weakened, at least when $n=3$. For an open set $W \subset X$, we call an $\left(\omega_{F}, T\right)$-compatible complex structure $J$ on $E \mid W\left(\omega_{F}, T\right)$-extendible along a collection of convergent sequences of regular points in $X$ if for each such sequence $x_{i} \rightarrow x$ with $x \in W$, the complex structures $T_{*} J$ on each $F_{x_{i}}$ (defined for all sufficiently large $i$ ) limit to an $\omega_{F}$-tame complex structure on $F_{x}$ (which is necessarily $\omega_{F^{-}}$ compatible and an extension of $T_{*} J$ on $\left.T\left(E_{x}\right) \subset F_{x}\right)$.

Addendum 3.3. If $n=3$ and there are critical points in $X-D$ that are not wrapped, fix a sequence of regular points converging to each unwrapped critical point in $X-D$, and assume that the given local complex structures on $E$ (including $\left.J_{C}\right)$ are $\left(\omega_{F}, T\right)$-extendible along these sequences. Then Lemma 3.2 still holds, where the structures $J$ comprising $\mathcal{J}$ are all required to be $\left(\omega_{F}, T\right)$-extendible along the given sequences, provided that in (c), the collar of $\partial C$ (if $D=C$ ) or of $\partial D($ if $D \neq C$ ) contains no unwrapped critical points.

Proof of Theorem 2.11. To prove (a), we wish to apply Lemma 3.2 to the hyperpencil $f: X-B \rightarrow \mathbb{C P}^{n-1}$. First, we show that the regular points of $f$ are dense in $X-B$ by the method previously used for Proposition 2.3. If any neighborhood $W \subset X-B$ consists entirely of critical points, choose $x_{0} \in W$ minimizing $\operatorname{dim} \operatorname{ker} d f_{x}$ and note that ker $d f$ is a smooth distribution near $x_{0}$. (As before we can realize it as ker $d(\pi \circ f)$ for a suitable projection $\pi$.) By integrating a vector field in ker $d f$, we obtain a curve of critical points in a single fiber, contradicting finiteness in Definition 2.4(3). Now we apply Lemma 3.2 to $X-B$, with $E=T(X-B), F=f^{*} T \mathbb{C P}^{n-1}, T=d f$ and $\omega_{F}=\omega_{\text {std }}$ (pulled back to $F$ ). We let $C=D \subset X-B$ consist of a closed, round ball (with center deleted) about each $b \in B$ in the local charts given by Definition 2.4(1), $U=V$ be corresponding punctured open balls, and $J_{C}$ be the corresponding complex structure on $E \mid U$. The required local complex structures over neighborhoods $W_{x}$ exist by Definition 2.4(2) at critical points $x$, and are easy to construct at regular points. By (a) of the lemma, we obtain the required almost-complex structure $J$ on $X-B$ (which immediately extends over $X$ ), using either the given definition of a 
hyperpencil or the variations of Remark 2.5(c). (For the last variation, set $D=X-B$.) Now (c) of the lemma (with the obvious radial collar of $\partial C$ ) gives contractibility of the space $\mathcal{J}_{C}$ of $\left(\omega_{\text {std }}, f\right)$-compatible almost-complex structures on $X$ extending $J_{C}$ on $C$ (with suitable $\left(\omega_{\text {std }}, d f\right.$ )-extendability or $f_{*} J$ fixed for the above variations). Let $\mathcal{J}=\bigcup_{C} \mathcal{J}_{C}$ be the space of all such $\left(\omega_{\text {std }}, f\right)$-compatible almost-complex structures on $X$ that are standard near $B$ (relative to fixed charts as in Definition 2.4(1), but on neighborhoods of variable size). Again fix $C$ and $U$ as above (with the fixed $f_{*} J$ standard on $U$ in the case of the last variation), and let $h_{t}: X \rightarrow X$ be a radial homotopy fixing $X-U$ with $h_{0}=\mathrm{id}_{X}$ and $h_{1}$ collapsing $C$ into $B$. Trivialize $T X \mid U$ in the obvious way, and extend $h_{t}$ to $\tilde{h}_{t}: T X \rightarrow T X$ as $h_{t} \times \operatorname{id}_{\mathbb{C}^{n}}$ over $U$ and $\operatorname{id}_{T X}$ elsewhere. Then pulling back induces a homotopy $H_{t}: \mathcal{J} \rightarrow \mathcal{J}$ with $H_{0}=\operatorname{id}_{\mathcal{J}}$ and $H_{1}(\mathcal{J}) \subset \mathcal{J}_{C}$. Composing with the previous contraction of $\mathcal{J}_{C}$ produces the required contraction of $\mathcal{J}$. A similar argument applies to the space of all $\left(\omega_{\text {std }}, f\right)$-compatible structures on $X$, since these must all agree on $T X \mid B$ by Lemma 4.4(b). The proof of (a) is now complete.

The existence part of (b) will follow from Theorem 3.1, so we begin by constructing suitable neighborhoods $W_{y}$ with forms $\eta_{y}$. Fix $C \subset U \subset X-B$ and $J$ on $X$ as above, and a neighborhood $W_{C}$ of $C$ in $X-B$ with closure cl $W_{C} \subset U$ a disjoint union of round balls. For each $y \in \mathbb{C P}^{n-1}$, let $K \subset F_{y}$ denote the (finite) subset of critical points of $f$ lying on the fiber $F_{y}$, and let $\Delta \subset X-U$ be a disjoint union of closed balls, one centered at each point of $K$. Define a closed 2-form $\sigma$ on $\Delta \cup U$ as follows: Choose $\sigma$ to tame $J$ on $T X \mid K$. For $\Delta$ sufficiently small, we can then assume $J$ is $\sigma$ tame on $\Delta$ (by openness of the taming condition). On $U$, take $\sigma$ to be the standard symplectic form from $\mathbb{C}^{n}$, in the local coordinates given by (1) of Definition 2.4, scaled so that its integral is $<1 / 2$ on each complex line through 0 intersected with $U$. Now $J$ is $\sigma$-tame on $\Delta \cup U$. Since $J$ is $\left(\omega_{\text {std }}, f\right)$ tame on $X-B, F_{y}-K$ is a smooth (noncompact) $J$-holomorphic curve in $X-K$ whose complex orientation agrees with its preimage orientation, and each component intersects $B$ nontrivially (by Definition 2.4(3)). To allow for the (presumably unlikely) possibility of $F_{y}$ being wildly knotted at $K$, we use the following trick: We can assume $\partial \Delta$ is transverse to $F_{y}$, so the two intersect in a finite collection of circles. Since each component of $F_{y}-K$ intersects $B$, we can connect each such circle to $B$ by a path in $F_{y}-K$. Let $\Delta_{0} \subset$ int $\Delta$ be a smaller collection of balls surrounding $K$, disjoint from these paths and with $\partial \Delta_{0}$ transverse to $F_{y}$. Then each component $F_{i}$ of the compact surface $F_{y}-\operatorname{int} \Delta_{0}$ either lies inside int $\Delta$ or intersects $B$. Let $W_{y}$ be the union of int $\Delta_{0} \cup W_{C}$ with a tubular neighborhood rel boundary of $F_{y}-\operatorname{int} \Delta_{0} \subset X-\operatorname{int} \Delta_{0}$. Extend each $F_{i}$ to a closed, oriented, smooth surface $\hat{F}_{i} \subset W_{y}$ by arbitrarily attaching a surface in $\Delta_{0}$. Then the classes $\left[\hat{F}_{i}\right] \in H_{2}\left(W_{y} ; \mathbb{Z}\right)$ form a basis. 
We now construct the required form $\eta_{y}$ on $W_{y}$ and apply Theorem 3.1. Since $F_{y}$ is $J$-holomorphic with $J \sigma$-tame on $\Delta \cup U, \sigma \mid F_{i} \cap\left(\Delta \cup W_{C}\right)$ is a positive area form. After rescaling $\sigma$ on $\Delta$ so that $\int_{\hat{F}_{i} \cap \Delta} \sigma<1 / 2$ for each $i$, we can extend $\sigma$ over each $F_{i}$ intersecting $B$ as a positive area form with $\int_{\hat{F}_{i}} \sigma=\# F_{i} \cap B$, the (positive) number of points of $B$ in $F_{i}$. Define $\pi: W_{y} \rightarrow W_{y}$ by smoothly splicing $\operatorname{id}_{W_{y} \cap(\Delta \cup U)}$ together with the normal bundle projection on $W_{y}-\Delta$ so that $\operatorname{Im} \pi \subset F_{y} \cup \Delta \cup U$ and $\pi \mid F_{y} \cup \Delta_{0} \cup W_{C}$ is the identity. Then $\eta_{y}=\pi^{*} \sigma$ is a well-defined closed 2-form on $W_{y}$. On $W_{y} \cap\left(\Delta \cup W_{C}\right.$ ) (away from $\left.\partial \Delta\right) \eta_{y}=\sigma$ tames $J$ (hence, each $J \mid$ ker $d f_{x}$ ) and $\eta_{y}$ is standard on $W_{C}$. Similarly, $\eta_{y} \mid\left(F_{y}-K\right)$ tames $J$ on each $T_{x} F_{y}=\operatorname{ker} d f_{x}$, so after narrowing the tubular neighborhood defining $W_{y}$, we can assume $\eta_{y}$ tames $J \mid$ ker $d f_{x}$ for all $x \in W_{y}$ (since the taming condition is open and the set of critical points of $f$ is closed). For each $F_{i}$ intersecting $B$, we have $\left\langle\eta_{y}, \hat{F}_{i}\right\rangle=\left\langle\sigma, \hat{F}_{i}\right\rangle=\# F_{i} \cap B=\left\langle c_{f}, \hat{F}_{i}\right\rangle$. (The last equality follows, e.g., by computing the intersection number of $\hat{F}_{i}$ (pushed slightly off $B$ ) with $f^{-1}\left(\mathbb{C P}^{n-2}\right) \subset X-B$. The only intersections occur in $U$, and each is +1 by the local description of $f$ there.) Similarly, for $F_{i}$ disjoint from $B$ we have $\left\langle c_{f}, \hat{F}_{i}\right\rangle=0=\left\langle\eta_{y}, \hat{F}_{i}\right\rangle$ since $\eta_{y}=\sigma$ is exact on the disjoint union of balls $\Delta$ containing $\hat{F}_{i}$. Thus $\left[\eta_{y}\right]=c_{f} \mid W_{y} \in H_{\mathrm{dR}}^{2}\left(W_{y}\right)$ since these agree on a basis of $H_{2}\left(W_{y} ; \mathbb{Z}\right)$. Now we can apply Theorem 3.1 to $X-B$ with $\omega_{Y}=\omega_{\text {std }}$ on $Y=\mathbb{C P}^{n-1}$ and $c=c_{f}$. Note that $W_{C}$ is a disjoint union of punctured open $2 n$-balls with $n \geq 2$, so $H_{\mathrm{dR}}^{1}\left(W_{C}\right)=0$. We obtain a closed 2-form $\eta$ on $X-B$ that is standard on $C$ (relative to the charts given in Definition 2.4(1)) and hence extends over $X$. Then $[\eta]=c_{f} \in H_{\mathrm{dR}}^{2}(X)$, and we can choose some $t>0$ so that $\omega_{t}=t \eta+f^{*} \omega_{\text {std }}$ tames $J$ on $X-B$.

Unfortunately, the form $\omega_{t}$ is singular at $B$. We verify this with a local model, and find a way to eliminate the singularities. In the given local coordinates at $b \in B, J$ is the standard complex structure on $\mathbb{C}^{n}, f$ is projectivization, and $\eta$ is the standard symplectic form. Up to a constant rescaling of the coordinates, the latter can be written in "complex spherical coordinates" (cf. [McS, Proposition 5.8]) as $\eta=r^{2} f^{*} \omega_{\text {std }}+\frac{1}{2 \pi} d\left(r^{2}\right) \wedge \beta$, where $r$ is the radial coordinate on $\mathbb{C}^{n}$ and $\beta$ is the pull-back to $\mathbb{C}^{n}$ $\{0\} \approx S^{2 n-1} \times \mathbb{R}$ of the connection 1-form on $S^{2 n-1}$ for the tautological bundle $S^{2 n-1} \rightarrow \mathbb{C P}^{n-1}$, whose corresponding horizontal distribution $H$ is orthogonal to the fibers. (To verify this formula, note that $H$ on $\mathbb{C}^{n}-\{0\}$ is orthogonal to each complex line $L$ through $0 \in \mathbb{C}^{n}$ under both the above $\eta$ and the standard symplectic form. Both descend from $S^{2 n-1}$ to $\omega_{\text {std }}$ on $\mathbb{C P}^{n-1}$ up to a constant scale factor, and both scale by $r^{2}$ radially, so they agree up to scale on $H$. On each $L, d\left(r^{2}\right) \wedge \beta=2 r d r \wedge d \theta$ is standard up to a scale factor independent of $L$. The two terms of $\eta$ are scaled compatibly since $d \eta=0$ by computation, using the fact that $d \beta$ pushes down to the tautological curvature form $2 \pi \omega_{\text {std }}$ on $\mathbb{C P}^{n-1}$ with Chern class $-\left[\omega_{\text {std }}\right]=$ 
-h.) Now $\omega_{t}(r)=\left(1+t r^{2}\right) f^{*} \omega_{\text {std }}+\frac{t}{2 \pi} d\left(r^{2}\right) \wedge \beta$ in these local coordinates. Clearly, this is singular at 0 since $\eta$ is nonzero there. However, the radial change of variables $R^{2}=\frac{1+t r^{2}}{1+t}\left(t\right.$ constant) shows that $\eta(R)=\frac{1}{1+t} \omega_{t}(r)$, so there is a radial symplectic embedding $\varphi:\left(\mathbb{C}^{n}-\{0\}, \frac{1}{1+t} \omega_{t}\right) \rightarrow\left(\mathbb{C}^{n}, \eta\right)$ sending any deleted neighborhood of 0 to a deleted neighborhood of the ball $R^{2} \leq \frac{1}{1+t}$. For $V \subset \mathbb{C}^{n}$ the image of the given coordinate chart at $b \in B$, define $\varphi_{0}: V \rightarrow \mathbb{C}^{n}$ to be a radially symmetric diffeomorphism onto an open ball, agreeing with $\varphi$ outside of a closed ball about 0 in $V$. Let $\omega$ be $\varphi_{0}^{*} \eta$ near each $b \in B$ and $\frac{1}{1+t} \omega_{t}$ elsewhere. These pieces fit together to define a symplectic form on $X$, since $\varphi$ is a symplectic embedding. (This construction is equivalent to blowing up $B$, applying Theorem 3.1 with $C=$ $\emptyset$ to the resulting singular fibration, and then blowing back down, but it avoids technical difficulties associated with taming on the blown up base locus.)

To complete the existence proof for (b), we only need to verify that the symplectic form $\omega$ on $X$ has the required properties. Away from $B$, we already know that $\omega=\frac{1}{1+t} \omega_{t}$ tames $J$. Near $b \in B$, we have local coordinates with $J$ standard and $\omega=\varphi_{0}^{*} \eta, \eta$ standard up to a constant scale factor. Since $\varphi_{0}$ is radially symmetric, it preserves the horizontal distribution $H$ on $\mathbb{C}^{n}-\{0\}$ and the form $\eta \mid H$ up to (nonconstant) scale. Also, $\varphi_{0}$ preserves each complex line through 0 , and $\varphi_{0}^{*} \eta$ is a positive area form on each. Since these complex lines and $H$ are $\eta$-orthogonal and $J$-holomorphic, $J$ is $\omega$-tame near $B$ and hence everywhere on $X$. To compute the cohomology class $[\omega] \in H_{\mathrm{dR}}^{2}(X)$, it suffices to work outside $C$. Then $[\omega]=\frac{1}{1+t}\left[\omega_{t}\right]=\frac{1}{1+t}\left(t c_{f}+\right.$ $\left.f^{*}\left[\omega_{\text {std }}\right]\right)=c_{f}$ since $\left[\omega_{\text {std }}\right]=h \in H_{\mathrm{dR}}^{2}\left(\mathbb{C P}^{n-1}\right)$. Thus, the constructed form $\omega$ has the required properties.

For fixed $J$, the space of taming $\omega$ with $[\omega]$ fixed is obviously convex; the rest of the theorem depends on the following lemma. This lemma allows us to extend a family of taming forms to a parametrized family of hyperpencils, starting from any subfamily with reasonable local topology.

Lemma 3.4. Let $f: X-B \rightarrow Y$ be a family of hyperpencils parametrized by a metrizable space $S$, and suppose the restriction of $f$ to some closed subset $S_{0} \subset S$ is $\omega_{0}$-tame for some continuous family $\omega_{0}$ of continuous 2-forms $\omega_{s}$ on the fibers $X_{s}, s \in S_{0}$. Suppose $S_{0}$ has a neighborhood $U_{0} \subset S$ with a $C^{0}$ retraction $r: U_{0} \rightarrow S_{0}$ covered by retractions $r_{X}: \pi_{X}^{-1}\left(U_{0}\right) \rightarrow \pi_{X}^{-1}\left(S_{0}\right)$ and $r_{Y}: \pi_{Y}^{-1}\left(U_{0}\right) \rightarrow \pi_{Y}^{-1}\left(S_{0}\right)$ with fiberwise derivatives $d r_{X}$ and $d r_{Y}$ continuous over $U_{0}, r_{Y}$ preserving $\omega_{\text {std }}, r_{X}$ a fiberwise diffeomorphism preserving $B$ and complex linear near $B$ with respect to the vector bundle structure, and $f \circ r_{X}=r_{Y} \circ f$. Then $\omega_{0}$ extends to a family $\omega$ parametrized by $S$, taming $f$. If $\omega_{0}$ consists of $C^{k}$-forms varying continuously in the $C^{k}$-topology, $(0 \leq$ $k \leq \infty)$, then $\omega$ inherits this property (provided $d r_{X}$ is $C^{k}$-continuous), 
and if the family $\omega_{0}$ is $C^{k}$ (for $\pi_{X}: X \rightarrow S$ a $C^{k+1}$-bundle map of $C^{k+1}$ manifolds) then so is $\omega$. Similarly, closure of each $\omega_{s}$ and the condition $\left[\omega_{s}\right]=c_{f} \in H_{\mathrm{dR}}^{2}\left(X_{s}\right)$ are inherited by $\omega$. We can also assume that $\omega$ tames a preassigned $J$ on $T^{v} X$ as specified in Lemma 2.10 (provided that $\omega_{0}$ tames $J$ over $\left.S_{0}\right)$, or that $\omega$ tames some $J$ for which $f_{*} J$ on $\operatorname{Im} d f \subset f^{*} T^{v} Y$ is preassigned, $r_{Y}$ invariant, standard near $B, \omega_{\text {std }}$-compatible, and induced from local complex structures on $T^{v} X$ as in Definition 2.9 (over $S_{0}$ ) and Definition 2.7(2) (over S).

Proof. If $J$ was not preassigned, construct it as follows: Find an $\omega_{0}$-tame $J_{0}$ over $S_{0}$ by Lemma 2.10 , and pull it back over $U_{0}$ by $r_{X}$. Then $J_{0}$ is continuous and $\left(\omega_{\text {std }}, f\right)$-compatible over $U_{0}$, standard near $B$ there, and if $f_{*} J$ is preassigned, it agrees with $f_{*} J_{0}$ there. Now $J_{0} \mid \pi_{X}^{-1}\left(S_{0}\right)$ extends to $J$ on $T^{v} X$ as specified in Lemma 2.10 (where $\omega_{0}$-tameness of $J$ only applies over $S_{0}$ ), by Lemma 3.2(a) applied to $X-B$ with $E=T^{v}(X-B)$, $F=f^{*} T^{v} Y, C=\pi_{X}^{-1}\left(S_{0}\right)$ union the closure of a suitable neighborhood of $B$, and $D$ equal to $C$ or $X$, depending on whether $f_{*} J$ was given. (Note that $X$ is metrizable since it is locally metrizable and paracompact Hausdorff, e.g., $[\mathbf{M u}, \S 42],[\mathbf{P}]$.) By the existence part of (b) of Theorem 2.11, each $X_{s}$, $s \in S-S_{0}$, has a smooth symplectic form $\omega_{s}$ taming $J \mid X_{s}$, with $\left[\omega_{s}\right]=c_{f}$. By openness of the taming condition and compactness of each fiber $X_{s}$, each $\omega_{s}$ extends over some neighborhood $W_{s}$ of $s \in S-S_{0}$ (e.g., via local triviality of $\pi_{X}$ ) so that it tames $J \mid \pi_{X}^{-1}\left(W_{s}\right)$. Similarly, each $s_{0} \in S_{0}$ has a neighborhood $W_{s_{0}}$ in $S$ over which $\omega_{0}$ extends as a taming form, preserving any additional conditions. (If only a continuous family is required, pull back by $r_{X}$. For a $C^{k}$-family of closed forms, $k \geq 1$, locally trivialize, $C^{k}$-extend the map $\left[\omega_{s}\right]: S_{0} \rightarrow H_{\mathrm{dR}}^{2}\left(X_{s}\right)$ over some $W_{s_{0}}$ (possibly with $\left[\omega_{s}\right]=c_{f}$ by hypothesis), find a $C^{k}$-family of representatives, and correct by $d \alpha$ for a suitably extended family of 1 -forms $\alpha$ to recover the original subfamily $\omega_{0}$.) Using a partition of unity on $S$ subordinate to the cover $\left\{W_{s} \mid s \in S\right\}$, splice together the local families $\omega_{s}$ into a global family $\omega$ extending $\omega_{0}$. The taming and closure conditions are preserved since each $\omega \mid X_{s}$ is a convex combination (with constant coefficients) of such forms. The lemma follows immediately.

To complete the proof of Theorem 2.11, first let $\omega_{s}, s=0,1$, be symplectic forms on $X$ associated to hyperpencils $f_{s}$ in a deformation class $\varphi$ as in (c). Then there is a deformation agreeing with $f_{0}$ and $f_{1}$, respectively, on neighborhoods of 0,1 in $I$. The above lemma, with $S=I$ and $S_{0}=\{0,1\}$, gives a smooth family of symplectic forms $\omega_{s}, 0 \leq s \leq 1$, interpolating between $\omega_{0}$ and $\omega_{1}$, with $\left[\omega_{s}\right]=c_{f_{0}}=c_{f_{1}}$ for each $s$. By Moser's Theorem $[\mathbf{M}]$, any deformation of cohomologous symplectic forms is realized by an isotopy, proving (c). A similar argument proves the last sentence of (b), completing the proof of the theorem: For fixed $k, 1 \leq k \leq \infty$, let $\mathcal{C}$ denote the $C^{k}$-space 
of all $C^{k}$-symplectic forms on $X$ as in (b) (for fixed $f$ and $f_{*} J$ ). Any continuous map $\varphi: \partial D^{m+1} \rightarrow \mathcal{C}\left(D^{m+1}\right.$ an $(m+1)$-disk $)$ can be interpreted as a $C^{k}$-continuous family $\omega_{0}$ of symplectic forms on $X$ parametrized by $\partial D^{m+1}$. Applying the above lemma to the constant family of hyperpencils on $X$ with $S=D^{m+1}, S_{0}=\partial D^{m+1}$, and the given $f_{*} J$ (which ensures that $f$ over $S_{0}$ is $\omega_{0}$-tame), we extend to a family parametrized by $D^{m+1}$, or equivalently a continuous map $D^{m+1} \rightarrow \mathcal{C}$ extending $\varphi$. This shows that $\mathcal{C}$ is weakly contractible $\left(\pi_{m}(\mathcal{C})=0\right.$ for all $\left.m\right)$. But $\mathcal{C}$ is an open subset of the affine subspace of 2-forms determining $c_{f} \in H_{\mathrm{dR}}^{2}(X)$, so it is a metrizable manifold of infinite dimension. In particular, it is an ANR, so weak contractibility implies contractibility $[\mathbf{P}]$. Since the $C^{\infty}$-forms are dense in the $C^{0}$-space of forms, and $\mathcal{C}$ extends to an open subset of a closed affine subspace of the latter, the required assertion follows from Theorem 16 of $[\mathbf{P}]$.

\section{Proofs of Lemmas 2.10 and 3.2}

The proofs of Lemmas 2.10 and 3.2 require a canonical method for interpolating between almost-complex structures. Our approach is a generalization of that of $[\mathbf{A B K L R}]$, Proposition 6.2. This depends on the $r=-\frac{1}{2}$ case of the following proposition, a similar form of which is stated without proof on p.100 of [ABKLR]. The proof below follows a suggestion of L. Sadun.

Proposition 4.1. For $r \in \mathbb{R}$, let $\rho_{0}: \mathbb{C}-(-\infty, 0] \rightarrow \mathbb{C}$ be the branch of $z^{r}$ with $\rho_{0}(1)=1$. Let $\mathcal{A} \subset \mathrm{GL}(m, \mathbb{C})$ be the open subset of matrices with no eigenvalues in $(-\infty, 0]$. Then there is a unique holomorphic map $\rho: \mathcal{A} \rightarrow \operatorname{GL}(m, \mathbb{C})$, which will be denoted by $\rho(A)=A^{r}$, such that each $\lambda$-eigenvector of $A$ is a $\rho_{0}(\lambda)$-eigenvector of $A^{r}$. This map has the following properties:

a) For $n \in \mathbb{Z}, A^{n}$ agrees with its usual meaning.

b) For $|r| \leq 1$ or $s \in \mathbb{Z},\left(A^{r}\right)^{s}=A^{\text {rs }}$.

c) If $T: \mathbb{C}^{m} \rightarrow \mathbb{C}^{k}$ is a linear transformation with $T A=B T$, then $T A^{r}=B^{r} T$ (whenever both sides are defined).

d) If $A$ is real, then so is $A^{r}$.

e) If $A$ is real and self-adjoint with respect to a given inner product $g$ on $\mathbb{R}^{m}$, then so is $A^{r}$.

f) For $n \in \mathbb{Z}-\{0\}, A^{1 / n}$ is the unique solution to the equation $X^{n}=A$ for which all eigenvalues of $X$ lie in $\operatorname{Im} \rho_{0}$.

Note that (d) and (c) imply that $\rho$ is canonically defined for any finitedimensional real vector space.

Proof. For any $A \in \mathcal{A}$, the Jordan form of $A$ splits $\mathbb{C}^{m}$ as the direct sum of the generalized eigenspaces $V_{\lambda}=\operatorname{ker}(A-\lambda I)^{m}$ ( $\lambda$ ranging over the eigenvalues of $A)$. On each $V_{\lambda}, A$ has the form $\lambda(I+N)$ for the nilpotent transformation $N=\frac{1}{\lambda} A-I$. Set $\rho(A)=\rho_{0}(\lambda) p(N)$ on $V_{\lambda}$, where $p(z)$ is the power 
series expansion about 0 of the function $\rho_{0}(1+z)=(1+z)^{r}$. (Note that $p(N)$ is a polynomial in the nilpotent transformation $N$.) On each $\lambda$ - eigenspace, $N=0$ and $A^{r}=\rho_{0}(\lambda) I$ as required. Properties (a) and (b) follow immediately from the corresponding properties for $\rho_{0}$ and $p$. Property (c) is also immediate, once we observe that the condition $T A=B T$ implies $T$ preserves or annihilates each generalized $\lambda$-eigenspace. For (d), take $A$ real and note that $\mathbb{R}^{m}$ is the span of the vectors $v+\bar{v}, v \in V_{\lambda}$ (where $\lambda$ ranges over all eigenvalues of $A$ ). Since $\bar{v} \in V_{\bar{\lambda}}, A^{r}(v+\bar{v})=A^{r} v+A^{r} \bar{v}=A^{r} v+\overline{A^{r} v}$, so this is real as required. Now (e) follows immediately from $g$-orthogonal diagonalizability. For (f), take any $X$ as given and write it as $\lambda(I+N)$ on each of its generalized eigenspaces $V_{\lambda}$. Then $A=X^{n}=\lambda^{n}(I+N)^{n}$ on each $V_{\lambda}$, and the last factor has the form $I+$ (nilpotent). Thus $A^{1 / n}=\rho\left(X^{n}\right)=X$, since $\lambda$ is the unique $n^{\text {th }}$ root of $\lambda^{n}$ in $\operatorname{Im} \rho_{0}$ and the power series for $\rho_{0}(1+z)$ inverts the exponentiation of $(I+N)^{n}$.

It remains to prove holomorphicity of $\rho$, from which uniqueness follows by density of diagonalizable matrices in $\mathcal{A}$. First note that the equation $\operatorname{det}(A-$ $\lambda I)=0$ in $(A, \lambda)$ exhibits the eigenvalues of matrices in $\mathcal{A}$ as an algebraic variety in $\mathcal{A} \times \mathbb{C}$. The subset $\mathcal{S} \subset \mathcal{A}$ of matrices failing to have $m$ distinct eigenvalues is then also a variety, and over $\mathcal{A}-\mathcal{S}$ the $m$ eigenvalues vary holomorphically. One can now locally construct holomorphically varying bases of eigenvectors over $\mathcal{A}-\mathcal{S}$, and in these bases, $\rho$ is easily seen to be holomorphic as required. To show that $\rho$ extends from $\mathcal{A}-\mathcal{S}$ to some holomorphic map $\hat{\rho}: \mathcal{A} \rightarrow \mathrm{GL}(m, \mathbb{C})$, note that nondiagonalizable matrices with $m-1$ distinct eigenvalues form a dense open subset of $\mathcal{S}$. Given such a matrix $A$, restrict it to its 2-dimensional generalized eigenspace $V_{\lambda}$, and note that in a suitable basis we obtain the matrix $A_{0}$ in the family

$$
A_{z}=\left(\begin{array}{cc}
\lambda+z & 0 \\
1 & \lambda
\end{array}\right), \text { with } \rho\left(A_{z}\right)=\left(\begin{array}{cc}
\rho_{0}(\lambda+z) & 0 \\
\frac{\rho_{0}(\lambda+z)-\rho_{0}(\lambda)}{z} & \rho_{0}(\lambda)
\end{array}\right)
$$

for small $z \neq 0$. (The latter equality is easy to verify using the eigenvectors $(0,1)$ and $(z, 1)$ for $\left.A_{z}.\right)$ Clearly, $\rho\left(A_{z}\right)$ extends holomorphically over $z=$ 0 . The Cauchy Integral Formula now provides the required holomorphic extension $\hat{\rho}$ on $\mathcal{A}$ (since the remaining subset of $\mathcal{S}$ has higher codimension in $\mathcal{A}$; cf. Hartogs' Theorem $[\mathbf{G H}])$.

Finally, we show $\hat{\rho}=\rho$. First, we assume $r=1 / n, n \in \mathbb{Z}-\{0\}$. For $A \in \mathcal{S}$, consider a sequence $\left(A_{i}\right)$ in $\mathcal{A}-\mathcal{S}$ converging to it. Then $\rho\left(A_{i}\right)=$ $\hat{\rho}\left(A_{i}\right) \rightarrow \hat{\rho}(A)$. By (a) and (b), $\left(\rho\left(A_{i}\right)\right)^{n}=A_{i}$; taking the limit shows that $(\hat{\rho}(A))^{n}=A$. Similarly, this sequence allows us to write each eigenvalue $\lambda$ of $\hat{\rho}(A)$ as $\lim \lambda_{i}$ for $\lambda_{i}$ an eigenvalue of $\rho\left(A_{i}\right)$. By the definition of $\rho$, $\lambda_{i} \in \operatorname{Im} \rho_{0}$, so $\lambda_{i}=\rho_{0}\left(\lambda_{i}^{n}\right)$ and $\lambda=\rho_{0}\left(\lambda^{n}\right)$. Now $\hat{\rho}(A)=\rho(A)$ by (f) as required. If $r=p / q \in \mathbb{Q}$, consider $A_{i} \rightarrow A$ as before. Then $\hat{\rho}\left(A_{i}\right)=\left(A_{i}^{1 / q}\right)^{p}$, 
so $\hat{\rho}(A)=\left(A^{1 / q}\right)^{p}=\rho(A)$ (by the previous case). The case of irrational $r$ now follows by continuity of $\rho$ and $\hat{\rho}$ with respect to $r$.

Corollary 4.2. For a real, finite-dimensional vector space $V$, let $\mathcal{B} \subset$ $\operatorname{Aut}(V)$ be the open set of linear operators with no real eigenvalues, and let $\mathcal{J} \subset \mathcal{B}$ denote the set of complex structures on $V$ (for both orientations of $V)$. Then there is a canonical real-analytic retraction $j: \mathcal{B} \rightarrow \mathcal{J}$. For any linear transformation $T: V \rightarrow W$ with $T A=B T$, we have $T j(A)=j(B) T$ (whenever both sides are defined).

Proof. We generalize $[\mathbf{A B K L R}]$. For $B \in \mathcal{B}$, note that $-B^{2}$ has no real eigenvalues $\leq 0$. (If $-\lambda^{2}$ were an eigenvalue of $-B^{2}$ with $\lambda \in \mathbb{R}$ then $\pm \lambda$ would be an eigenvalue of $B$, since $0=\operatorname{det}\left(-B^{2}+\lambda^{2} I\right)= \pm \operatorname{det}(B+$ $\lambda I) \operatorname{det}(B-\lambda I)$.) Thus, we can define $j(B)$ to be $B\left(-B^{2}\right)^{-1 / 2}$. The two factors commute by Proposition $4.1(\mathrm{c})$, so $(j(B))^{2}=-I$ as required. For $J \in \mathcal{J}, j(J)=J$. The rest of the corollary also follows immediately.

Now fix $J_{1}, \ldots, J_{k} \in \mathcal{J}$ and let $t=\left(t_{1}, \ldots, t_{k}\right)$ vary over the simplex $\sum t_{i}=1$, each $t_{i} \geq 0$. Suppose each $B_{t}=\sum t_{i} J_{i}$ has no real eigenvalues. For example, this is guaranteed if $J_{1}, \ldots, J_{k}$ are all $\omega$-tame for a fixed $\omega$ on $V$ (since for $v \neq 0, \omega\left(v, B_{t} v\right)=\sum t_{i} \omega\left(v, J_{i} v\right)>0$ but $\left.\omega(v, v)=0\right)$. Then we obtain an analytic simplex $j\left(B_{t}\right)$ of complex structures (complex simplex?) with vertices $J_{1}, \ldots, J_{k}$. We show that if the vertices are $\omega$ compatible for a fixed $\omega$ on $V$, then so is each $j\left(B_{t}\right)$ (cf. [ABKLR]): Compatibility implies that the bilinear form $g_{i}(v, w)=\omega\left(v, J_{i} w\right)$ is positive definite and symmetric for each $i$, as is the form $g=\sum t_{i} g_{i}$. Since $g(v, w)=\omega\left(v, B_{t} w\right)$, we have $g\left(B_{t} v, w\right)=\omega\left(B_{t} v, B_{t} w\right)=-\omega\left(B_{t} w, B_{t} v\right)=$ $-g\left(B_{t} w, v\right)=-g\left(v, B_{t} w\right)$. Thus $B_{t}$ is skew-adjoint with respect to the inner product $g$, so $-B_{t}^{2}$ and $\left(-B_{t}^{2}\right)^{-1 / 2}$ are self-adjoint (the latter by Proposition 4.1(e)). Hence $j\left(B_{t}\right)$ is skew-adjoint, implying that $\omega\left(j\left(B_{t}\right) v, j\left(B_{t}\right) w\right)=$ $g\left(j\left(B_{t}\right) v, B_{t}^{-1} j\left(B_{t}\right) w\right)=g\left(v, B_{t}^{-1} w\right)=\omega(v, w)$ as required. Furthermore, $\omega\left(v, j\left(B_{t}\right) v\right)=\omega\left(v, B_{t}\left(-B_{t}^{2}\right)^{-1 / 2} v\right)=g\left(v,\left(-B_{t}^{2}\right)^{-1 / 2} v\right)>0$ for $v \neq 0$, since $-B_{t}^{2}$ and hence $\left(-B_{t}^{2}\right)^{-1 / 2}$ are $g$-orthogonally diagonalizable with all eigenvalues positive.

Question 4.3. If $J_{1}, \ldots, J_{k}$ are only given to be $\omega$-tame (for a fixed $\omega$ ), is each $j\left(B_{t}\right) \omega$-tame?

An affirmative answer would allow us to replace compatibility by taming throughout the paper, define $\omega$-tame hyperpencils in a purely local way, remove $f_{*} J$ from the statement of the Main Theorem 2.11(b), and significantly simplify some of the proofs. For example, (b) of Lemma 3.2 with $D=C$ would follow immediately from the proof of (a), rendering the case of the lemma with $D \neq C$ unnecessary, along with the nonlocal condition on $f_{*} J_{\alpha}$ in the definition of $\omega$-tame hyperpencils, and the fixed $f_{*} J$ in Theorem $2.11(\mathrm{~b})$. 
Proof of Lemma 2.10. The main difficulty is that Lemma 3.2(b) does not apply directly at the base locus. For an $\omega$-tame hyperpencil $f: X-B \rightarrow$ $\mathbb{C P}^{n-1}$ and $b \in B$, Definition 2.9 gives a neighborhood $W_{b}$ of $b$ in $X$ with an $\omega$-tame $J_{b}$ on $W_{b}$ for which $J_{b} \mid W_{b}-\{b\}$ is $\left(\omega_{\text {std }}, f\right)$-compatible. If $f_{*} J_{b}$ is not standard near $b$, we must correct this by a perturbation. First note that $J_{b} \mid T_{b} X$ must agree with the standard structure $i$, by (b) of the following lemma, which is proved below.

Lemma 4.4. (a) A linear complex structure $J$ on $\mathbb{R}^{2 n}, n \neq 1$, is determined by its 1-dimensional (oriented) complex subspaces.

(b) If $f: \mathbb{C}^{n}-\{0\} \rightarrow \mathbb{C P}^{n-1}$ denotes projectivization, $n \geq 2$, and $J$ is a continuous (positively oriented) almost-complex structure on a neighborhood $W$ of 0 in $\mathbb{C}^{n}$, with $J \mid W-\{0\}\left(\omega_{\text {std }}, f\right)$-tame, then $J \mid T_{0} \mathbb{C}^{n}$ is the standard complex structure.

Thus there is a neighborhood $W$ of $b$ in $W_{b}$ for which the operators $A_{t}=$ $(1-t) J_{b}+t i, 0 \leq t \leq 1$, have no real eigenvalues, and for which the resulting complex structures $j\left(A_{t}\right)$ from Corollary 4.2 are $\omega$-tame. Since $f_{*} j\left(A_{t}\right)=j\left((1-t) f_{*} J_{b}+t f_{*} i\right)$ by the naturality statement in Corollary 4.2, the paragraph following the proof of that corollary shows that each $j\left(A_{t}\right)$ on $W-\{b\}$ is $\left(\omega_{\text {std }}, f\right)$-compatible. Let $\rho: X \rightarrow[0,1]$ be a continuous function with support in $W$ and identically 1 near $b$. Then we can add $\left(W, j\left(A_{\rho}\right)\right)$ to the open cover $\left\{W_{\alpha}\right\}$ and replace each $W_{\alpha}$ by $W_{\alpha}-\operatorname{supp} \rho$, recovering the hypotheses of the lemma, with the unique structure near $b$ standard. After applying this procedure when necessary to each $b \in B$, we invoke Lemma 3.2(b), with $E=T(X-B), F=f^{*} T \mathbb{C P}^{n-1}, T=d f$, and $C$ equal to the closure of a sufficiently small neighborhood of $B$ so that we can let $J_{C}$ be the standard structure $i$ near each $b \in B$. The resulting structure on $X-B$ extends in the obvious way over $B$, completing the proof for a single hyperpencil.

A similar argument applies to families over metrizable parameter spaces. Cover $B \subset X$ by neighborhoods $W$ as before, and choose $\rho: X \rightarrow[0,1]$ supported in their union. The resulting forms $f_{*} j\left(A_{\rho}\right)$ fit together as required, and Lemma 3.2(b) again completes the proof.

Proof of Lemma 4.4. To reduce (b) to (a), note that each complex line through 0 (with respect to the standard complex structure $i$ on $\mathbb{C}^{n}$ ) intersects $W$ in a $J$-complex curve (with the same orientation), since its tangent spaces away from 0 are given by ker $d f$ and $J$ is $\left(\omega_{\text {std }}, f\right)$-tame. ( $J$-complexity extends over 0 by continuity.) Thus, the $i$-complex lines through 0 in $T_{0} \mathbb{C}^{n}$ are also $J$-complex lines. To prove (a), assume each 1 -dimensional $i$-linear subspace of $\mathbb{C}^{n}=\mathbb{R}^{2 n}$ is $J$-linear, pick two $i$-linearly independent vectors $v, w \in \mathbb{C}^{n}$ and let $W=\operatorname{span}_{i} w$ and $V_{\lambda}=\operatorname{span}_{i}(v+\lambda w)$ for $\lambda \in \mathbb{C}$. These subspaces are both $i$-complex and $J$-complex lines. Thus, the projections of $V_{\lambda} \subset V_{0} \oplus W$ to $V_{0}$ and $W$ determine a map $\varphi_{\lambda}: V_{0} \rightarrow W$ (whose graph is 
$\left.V_{\lambda}\right)$ that is both $i$-linear and $J$-linear, and is determined by the condition $\varphi_{\lambda}(v)=\lambda w$. Now $\psi=\varphi_{1}^{-1} \circ \varphi_{i}: V_{0} \rightarrow V_{0}$ is both $i$ - and $J$-linear and given by $\psi(v)=i v$, so $\psi$ is multiplication by $i$. It follows that $J$ commutes with $i$ on the complex line $V_{0}$, so that these two complex structures on $V_{0}$ agree. But $V_{0}$ was chosen arbitrarily, so $J=i$ everywhere.

Proof of Lemma 3.2 and Addendum 3.3. We begin proving (a) with a partition of unity argument that works outside of $D-C$. Cover the space $X$ by open sets $W_{\alpha}$ with complex structures $J_{\alpha}$ on $E \mid W_{\alpha}$ as in the lemma. We can assume that $U$ is the unique $W_{\alpha}$ intersecting $C$ (with corresponding $\left.J_{\alpha}=J_{C}\right)$, and that any $W_{\alpha}$ intersecting $D$ lies in $V$, so that $T_{*} J_{\alpha}=J_{D} \mid W_{\alpha}$ there. Since $X$ is metrizable, it is paracompact by Stone's Theorem (e.g., [MS], [Mu, Theorem 41.4]), so there is a partition of unity $\left\{\rho_{\alpha}\right\}$ subordinate to the covering $\left\{W_{\alpha}\right\}$. Let $A=\sum \rho_{\alpha} J_{\alpha}: E \rightarrow E$. To make this into a complex structure by the retraction in Corollary 4.2 , we must investigate where $A$ could have real eigenvalues. First note that the map $B=\sum \rho_{\alpha} T_{*} J_{\alpha}: T(E) \rightarrow T(E)$ has no real eigenvalues on any fiber since each $T_{*} J_{\alpha}$ is $\omega_{F}$-tame. Since $T A=B T$, each $\lambda$-eigenvector of $A$ is mapped by $T$ to 0 or a $\lambda$-eigenvector of $B$, so any real eigenvector of $A$ must lie in $\operatorname{ker} T$.

To rule out real eigenvectors in $\operatorname{ker} T$, recall the subset $P \subset \operatorname{ker} T \subset E$ introduced for Definition 2.2 (of wrapped points). For each $x \in X$, any $v \in P_{x}$ can be written as $\lim v_{i}$ with $v_{i} \in \operatorname{ker} T_{x_{i}}$ for some sequence $\left(x_{i}\right)$ of regular points converging to $x$. After passing to a subsequence, we can assume the oriented 2-planes $\operatorname{ker} T_{x_{i}}$ (in the preimage orientation) converge to an oriented 2-plane $\Pi \subset P_{x}$ containing $v$. For each $J_{\alpha}$ defined on $E_{x}$, each $\operatorname{ker} T_{x_{i}}$ ( $i$ large) will be $J_{\alpha}$-complex (compatibly oriented) as will the limit $\Pi$. It is now easy to construct a decomposition $\operatorname{span}_{\mathbb{R}} P_{x}=\bigoplus \Pi_{j}$, where each oriented real 2-plane $\Pi_{j}$ is a $J_{\alpha}$-complex line for each $J_{\alpha}$ defined at $x$. Clearly, the quotient $Q_{x}=\operatorname{ker} T_{x} / \operatorname{span}_{\mathbb{R}} P_{x}$ inherits a complex structure $\bar{J}_{\alpha}$ from each such $J_{\alpha}$, and these are all compatible with the same orientation on $Q_{x}$ (inherited via ker $T_{x}$ from $\omega_{F} \mid T\left(E_{x}\right)$ ). If $x$ is wrapped, then $\operatorname{dim}_{\mathbb{C}} Q_{x} \leq 1$, so each $\bar{J}_{\alpha}$ is $\omega$-tame for some fixed $\omega$ on $Q_{x}$. It follows as before that $\sum \rho_{\alpha}(x) \bar{J}_{\alpha}$ has no real eigenvalues on $Q_{x}$, so any real eigenvector of $A_{x}$ lies in $\operatorname{span}_{\mathbb{R}} P_{x}=\bigoplus \Pi_{j}$. But a direct sum $\omega$ on this space tames each $J_{\alpha}$, so we conclude that $A_{x}$ has no real eigenvalues when $x$ is wrapped. If $x \in X-D$ is not wrapped (Addendum 3.3), then by hypothesis $n=3$, and $\operatorname{dim}_{\mathbb{C}} Q_{x} \geq 2$, so $T_{x}=0$ (since density of regular points implies $\operatorname{dim}_{\mathbb{C}} \operatorname{span}_{\mathbb{R}} P_{x} \geq 1$ ). We are also given a sequence $x_{i} \rightarrow x$ of regular points along which each $J_{\alpha}$ is $\left(\omega_{F}, T\right)$-extendible, so each relevant $T_{*} J_{\alpha}$ extends continuously and $\omega_{F^{-}}$ compatibly to $F \mid\left\{x_{i}\right\} \cup\{x\}$. If we pass to a suitable subsequence, $\frac{T_{x_{i}}}{\left\|T_{x_{i}}\right\|}$ will converge to a nonzero transformation that is complex linear for each relevant $\alpha$. Then over the subspace $\left\{x_{i}\right\} \cup\{x\}, \frac{T}{\|T\|}$ will have a wrapped 
point at $x$, and each relevant $J_{\alpha}$ will be $\left(\omega_{F}, \frac{T}{\|T\|}\right)$-compatible, so the previous argument again shows that $A_{x}$ has no real eigenvalues. Thus, $A$ can have real eigenvalues only at unwrapped critical points in $D$.

Corollary 4.2 provides a complex structure $j(A)$ that is well-defined and continuous except where $A$ has real eigenvalues. By construction, $j(A)=J_{C}$ on some neighborhood of $C$, and $T_{*} j(A)=j(B)$, which is defined everywhere and equals $J_{D}$ on a neighborhood $V^{\prime}$ of $D$. Furthermore $j(A)$ is $\left(\omega_{F}, T\right)$ compatible (in particular, $\left(\omega_{F}, T\right)$-tame) since each $T_{*} J_{\alpha}$, and hence $j(B)$, is $\omega_{F}$-compatible. (This is the only place where we require compatibility instead of taming; cf. Question 4.3. Our use of compatibility in proving Lemma 2.10 can be avoided using openness of taming, cf. [G3, second paragraph following Addendum 2.6].) Similarly, in the case of Addendum 3.3, $j(A) \mid X-D$ is $\left(\omega_{F}, T\right)$-extendible along the given sequences, as is any structure mapping to $j(B)$. This proves (a) of Lemma 3.2 (and its addendum) when $D=C$. The general case reduces to the case $D=X$ of the lemma after we intersect each $W_{\alpha}$ with $V^{\prime}$, add a new $W_{\alpha}=X-D$ with $J_{\alpha}=j(A) \mid X-D$, and extend $J_{D}$ to $j(B): T(E) \rightarrow T(E)$.

We now complete the proof of (a) in the remaining case $D=X$, while simultaneously preparing for (b). Recall that we just showed each $v \in P$ lies in an oriented 2-plane $\Pi=\lim \operatorname{ker} T_{x_{i}} \subset P_{x}$, for some sequence $\left(x_{i}\right)$ of regular points converging to $x$, and that $\Pi$ is a complex line for each $J_{\alpha}$ (including $J_{C}$ ) defined on $E_{x}$. Each such $J_{\alpha}$ now induces a complex structure $J_{\Pi}$ on the quotient $E_{x} / \Pi$, and this structure is the limit of the corresponding structures on $E_{x_{i}} / \operatorname{ker} T_{x_{i}}$. Since these latter structures are also determined by $J_{D}$ on $T\left(E_{x_{i}}\right)$ via the isomorphism induced by $T_{x_{i}}$, they are independent of $\alpha$, as is $J_{\Pi}$. If $Z \subset X$ denotes the subset of $X$ for which $\operatorname{dim}_{\mathbb{C}} \operatorname{span}_{\mathbb{R}} P_{x} \geq 2$, then each $E_{x}$ with $x \in Z$ contains at least two such distinct planes $\Pi_{1}$ and $\Pi_{2}$, and the $J_{\alpha}$-complex monomorphism $E_{x} \hookrightarrow E_{x} / \Pi_{1} \oplus E_{x} / \Pi_{2}$ shows that the structures $J_{\alpha}$ all agree on $E_{x}$. Thus, over $C^{\prime}=C \cup \operatorname{cl} Z$, the structures $J_{\alpha} \mid W_{\alpha}-C$ and $J_{C}$ all fit together into a continuous $\left(\omega_{F}, T\right)$-compatible structure $J_{C^{\prime}}$ on $E \mid C^{\prime}$ with $T_{*} J_{C^{\prime}}=J_{D} \mid C^{\prime}$, depending only on $J_{C}$ and $J_{D}$. We can now construct a $J_{C^{\prime}}$-Hermitian fiber metric on $E \mid C^{\prime}$ whose real part extends to a fiber metric $g$ on $E$ (using partitions of unity and the Tietze Extension Theorem). Over $X-C^{\prime}, P$ is an oriented (real) 2-dimensional subbundle of $E$. (To verify that $P \mid X-C^{\prime}$ is a continuous section of the Grassmann bundle, note that for $x \in X-C^{\prime}$ and any neighborhood $V_{\Pi}$ of the plane $\Pi=P_{x}$, there is a neighborhood $U_{x}$ of $x$ in $X$ on which all regular points map into $V_{\Pi}$; otherwise one could construct a sequence $x_{i} \rightarrow x$ with $\operatorname{ker} T_{x_{i}}$ converging to a plane $\neq \Pi$. Now $U_{x}-C^{\prime}$ maps into the closure of $V_{\Pi}$.) Using the given orientation of $P \mid X-C^{\prime}$ and the metric $g$, define $J \mid X-C^{\prime}$ to be counterclockwise $\frac{\pi}{2}$-rotation on $P$ and the structure determined by $J_{P_{x}}$ on each $P_{x}^{\perp} \cong E_{x} / P_{x}$. (Note that $J_{P_{x}}$ is continuous in $x$ since it is locally induced by the structures $J_{\alpha}$.) For each $x \in X-C^{\prime}, T_{x}$ factors through 
$E_{x} / P_{x}$ on which $J$ and $J_{\alpha}$ agree, so $J \mid X-C^{\prime}$ is $\left(\omega_{F}, T\right)$-compatible, with $T_{*} J\left|X-C^{\prime}=J_{D}\right| X-C^{\prime}$. Thus the proof of (a) is completed by showing that $J \mid X-C^{\prime}$ and $J_{C^{\prime}}$ fit together continuously at each $x \in C^{\prime}$. If this fails, then there is a sequence $x_{i} \rightarrow x$ for which each $J_{x_{i}}$ lies outside a fixed neighborhood of $J_{x}$ in the bundle $\operatorname{End}(E)$. By continuity of $J_{C^{\prime}}$, we can pass to a subsequence $\left(x_{i}\right)$ lying in $X-C^{\prime}$, and then further assume the oriented 2-planes $P_{x_{i}}$ converge to some $\Pi$ at $x$ by compactness of the Grassmann manifold. Since $g$ is continuous and Hermitian at $x$, it is now routine to obtain the contradiction that $J_{x_{i}} \rightarrow J_{x}$. (Compare with a fixed $J_{\alpha}$ on the given orthogonal summands.)

To prove (b) of the lemma, we introduce a 2 -form $\omega_{E}$ on $E$ that is assumed to tame each $J_{\alpha}$ (including $J_{C}$ ). On $X-C^{\prime}$, let $Q$ denote the $\omega_{E^{-}}$-orthogonal complement of the 2-dimensional (real) oriented subbundle $P \subset E \mid X-$ $C^{\prime}$. Since each $P_{x}$ is a complex line for some $\omega_{E}$-tame $J_{\alpha}$ on $E_{x}, \omega_{E}$ is nondegenerate (and positively oriented) on $P$. Thus $E \mid X-C^{\prime}=Q \oplus P$ is an $\omega_{E}$-orthogonal direct sum splitting. Now any subbundle of $E \mid X-C^{\prime}$ complementary to $P$ can be written as graph $\psi$ for some continuous section $\psi$ of $\operatorname{Hom}(Q, P)$. For $J$ as constructed in the previous paragraph, let $J_{\psi}$ denote the complex structure on $E \mid X-C^{\prime}$ given by $J$ on $P$ and by $J_{P_{x}}$ on each graph $\psi_{x} \cong E_{x} / P_{x}$. To express any $J_{\psi}$ in terms of $J_{0}$ (which is given by $J_{P_{x}}$ on each $\left.Q_{x}\right)$, note that these agree on the quotient $E_{x} / P_{x}$, so for any $(q, \psi(q)) \in$ graph $\psi \subset Q \oplus P$ we have $J_{\psi}(q, \psi(q))=\left(J_{0} q, \psi\left(J_{0} q\right)\right)$. Then for any $(q, p) \in Q \oplus P$ we have $J_{\psi}(q, p)=J_{\psi}(q, \psi(q))+J_{\psi}(0,-\psi(q)+p)=$ $\left(J_{0} q,\left(\psi J_{0}-J \psi\right) q+J p\right)$. In particular,

$$
\omega_{E}\left((q, p), J_{\psi}(q, p)\right)=\omega_{E}\left(q, J_{0} q\right)+\omega_{E}(p, J p)+\omega_{E}\left(p,\left(\psi J_{0}-J \psi\right) q\right) .
$$

Note that $J$ is $\omega_{E}$-tame on $P \mid X-C^{\prime}$ since the fibers are correctly oriented $J$-complex lines. In the next paragraph, we will show that $J_{0}$ is $\omega_{E}$-tame on $Q$, so the first two terms on the right side of Equation (4.1) have positive sum whenever $(q, p) \neq(0,0)$. Now choose $\psi$ so that graph $\psi$ is a $J$-complex subbundle of $E \mid X-C^{\prime}$. Then $J_{\psi}=J \mid X-C^{\prime}$. At each $x \in C^{\prime}, J$ agrees with some $J_{\alpha}$, so it is $\omega_{E^{-}}$-tame on $E_{x}$. By openness of the taming condition, we conclude that $J$ is $\omega_{E}$-tame over some neighborhood $U^{\prime}$ of $C^{\prime}$. Thus, the left side of (4.1) is positive for $x \in U^{\prime}-C^{\prime},(q, p) \neq(0,0)$. Replacing $\psi$ by $\rho \psi$ in (4.1) for any $\rho: U^{\prime}-C^{\prime} \rightarrow[0,1]$, we obtain a convex combination of two positive quantities, showing that $J_{\rho \psi}$ is $\omega_{E}$-tame on $E \mid U^{\prime}-C^{\prime}$. If we choose $\rho: X-C^{\prime} \rightarrow[0,1]$ to be 1 near $C^{\prime}$ and 0 outside $U^{\prime}$, then $J_{\rho \psi}$ extends over $C^{\prime}$ as $J$, providing the required $\omega_{E}$-tame complex structure on $E$.

We finish the proof of (b) by showing that $J_{0} \mid Q_{x}$ is $\omega_{E}$-tame for all $x \in X-C^{\prime}$. If $g$ denotes the $J_{0}$-invariant, symmetric bilinear form on $Q_{x}$ given by $g(v, w)=\frac{1}{2}\left(\omega_{E}\left(v, J_{0} w\right)+\omega_{E}\left(w, J_{0} v\right)\right)$, then $g(q, q)=\omega_{E}\left(q, J_{0} q\right)$, so it suffices to show $g$ is positive definite. For a fixed $J_{0}$-invariant inner product on $Q_{x}$, let $Q_{-} \subset Q_{x}$ be the span of all nonpositive eigenvectors of 
$g$. By $J_{0}$-invariance of $g$ and the background inner product, $Q_{-}$is a $J_{0^{-}}$ complex subspace of $Q_{x}$. Let $\psi: Q_{x} \rightarrow P_{x}$ be a (real) linear transformation whose graph is a $J_{\alpha}$-complex subspace of $E_{x}=Q_{x} \oplus P_{x}$ for some $\alpha$. Then $J_{\psi} \mid$ graph $\psi=J_{\alpha} \mid$ graph $\psi$ is $\omega_{E}$-tame. Thus (4.1) is positive for all nonzero $(q, p)$ with $p=\psi(q)$. The latter condition cancels two terms, so we obtain $\omega_{E}\left(q, J_{0} q\right)+\omega_{E}\left(p, \psi\left(J_{0} q\right)\right)>0$. Since the first term is $g(q, q)$, it is nonpositive on $Q_{-}$, implying that $p=\psi(q)$ cannot vanish on $Q_{-}$unless $q$ does. Hence, $\psi \mid Q_{-}$is a monomorphism and $\operatorname{dim}_{\mathbb{C}} Q_{-} \leq \operatorname{dim}_{\mathbb{C}} P_{x}=1$. It now suffices to rule out the case $\operatorname{dim}_{\mathbb{C}} Q_{-}=1$. In this case, note that $J_{\alpha}$ and $\omega_{E}$ induce the same orientation on $E_{x}$ and on $P_{x}$, hence, on $Q_{x} \cong E_{x} / P_{x}$ (by $\omega_{E}$-orthogonality of the splitting $E_{x}=Q_{x} \oplus P_{x}$ ). If $\operatorname{dim}_{\mathbb{C}} Q_{x}=1$, this implies $J_{0} \mid Q_{x}$ is $\omega_{E}$-tame, so it suffices to assume $\operatorname{dim}_{\mathbb{C}} Q_{x}>1$. Then the function $q \mapsto g(q, q)$ realizes both positive and nonpositive values on $Q_{x}-\{0\}$, so by connectedness there is a nonzero $q \in Q_{x}$ with $g(q, q)=0$. It follows that $\omega_{E}$ vanishes on the $J_{0}$-complex line $Q_{0} \subset Q_{x}$ containing $q$, so it is degenerate on the $\omega_{E}$-orthogonal sum $Q_{0} \oplus P_{x} \subset E_{x}$. But this latter subspace is $J_{\alpha}$-complex since it projects to a $J_{P_{x}}$-complex line in $E_{x} / P_{x}$, contradicting the hypothesis that $J_{\alpha}$ is $\omega_{E}$-tame.

To prove (c), assume $X_{0}=X-\operatorname{int} C$ is locally compact, and let $\mathcal{C}(\xi)$ be the space of (continuous) sections of the bundle $\xi=\operatorname{End}\left(E \mid X_{0}\right)$ of endomorphisms $E_{x} \rightarrow E_{x}\left(x \in X_{0}\right)$ in the compact-open topology. A subbasis for this topology is given by all subsets of the form $S(B, W)=\{\sigma \in \mathcal{C}(\xi) \mid \sigma(B) \subset$ $W\} \subset \mathcal{C}(\xi)$, for $B \subset X_{0}$ compact and $W \subset \xi$ open. (See, e.g., [Mu] for the space of all maps $X_{0} \rightarrow \xi$, then restrict to the subspace of sections $\mathcal{C}(\xi)$.) We need three basic facts about this topology: (1) The evaluation map $e: X_{0} \times \mathcal{C}(\xi) \rightarrow \xi$ (by $e(x, \sigma)=\sigma(x)$ ) is continuous. (This follows easily from local compactness.) (2) If $S$ is any topological space and $\pi_{X_{0}}: X_{0} \times S \rightarrow X_{0}$ is projection, then any section of the bundle $\pi_{X_{0}}^{*} \xi=\xi \times S \rightarrow X_{0} \times S$ has the form $\left(\tau, \pi_{S}\right)$ where $\tau: X_{0} \times S \rightarrow \xi$ can be interpreted as a map $S \rightarrow \mathcal{C}(\xi)$. This correspondence gives a bijection between continuous sections of $\pi_{X_{0}}^{*} \xi$ and continuous maps $S \rightarrow \mathcal{C}(\xi)$ (cf. [Mu]). (3) If $X_{0}$ is compact, then the compact-open topology on $\mathcal{C}(\xi)$ equals the $C^{0}$-topology induced by any fiber metric on $\xi$. (This is clear once we observe that subtracting a section of $\xi$ induces a fiberwise-isometric homeomorphism of $\xi$, so it suffices to compare neighborhoods of the 0 -section.)

In either case (a) or (b), let $\mathcal{J} \subset \mathcal{C}(\xi)$ be the subspace consisting of all complex structures on $E \mid X_{0}$ satisfying the conclusion of the lemma - that is, $\left(\omega_{F}, T\right)$-compatible extensions $J$ of $J_{C} \mid \partial C$ with $T_{*} J=J_{D}$ on $D \cap X_{0}$, and with $J \omega_{E}$-tame in case (b) or (for the addendum) $\left(\omega_{F}, T\right)$-extendible along the given sequences. For $X_{0}$ compact, the first step in showing the contractibility of $\mathcal{J}$ is to apply the lemma to the metrizable space $\tilde{X}=$ $I \times X_{0} \times \mathcal{J}$, with the structures $\tilde{E}, \tilde{F}, \tilde{T}, \omega_{\tilde{F}}\left(\right.$ and $\omega_{\tilde{E}}$ in case (b)) pulled 
back in the obvious way by projection $\pi_{X_{0}}: \tilde{X} \rightarrow X_{0}$. We set $\tilde{C}=(\{0,1\} \times$ $\left.X_{0} \cup I \times \partial C\right) \times \mathcal{J}$ and $\tilde{D}=\left(\{0,1\} \times X_{0} \cup I \times\left(D \cap X_{0}\right)\right) \times \mathcal{J}$. To define $J_{\tilde{C}}$ on a neighborhood $\tilde{U}$ of $\tilde{C}$, first consider the tautological complex structure $J_{\text {taut }}=\left(e, \pi_{\mathcal{J}}\right): X_{0} \times \mathcal{J} \rightarrow \xi \times \mathcal{J}=\pi_{X_{0}}^{*} \xi=\operatorname{End}\left(\tilde{E} \mid\{0\} \times X_{0} \times \mathcal{J}\right)$. Let $h:\left[0, \frac{1}{2}\right] \times I \rightarrow I$ be continuous with $h(0, t)=t, h(s, 1)=1$ and $h(s, t)=0$ for $s \geq t$. Extend to a map $\left[0, \frac{1}{2}\right] \times K \rightarrow K$, for the collar $K \approx I \times \partial C$ in $X_{0}$, by $\left(h \circ\left(\operatorname{id}_{[0,1 / 2]} \times \pi_{I}\right), \pi_{\partial C}\right)$ (so the $\partial C$ factor is carried along trivially), and let $H:\left[0, \frac{1}{2}\right] \times X_{0} \rightarrow X_{0}$ be the resulting extension by $\pi_{X_{0}}$ outside of $K$. Compatibility of the collar neighborhood shows that $\left(H \times \operatorname{id}_{\mathcal{J}}\right)^{*}\left(\tilde{E} \mid\{0\} \times X_{0} \times \mathcal{J}\right)$ can be identified with $\tilde{E} \mid\left(\left[0, \frac{1}{2}\right] \times X_{0} \times \mathcal{J}\right)$, and similarly for $\tilde{F}, \omega_{\tilde{F}}, \tilde{T}$ (and $\omega_{\tilde{E}}$ and $J_{\tilde{D}}=\pi_{X_{0}}^{*}\left(J_{D} \mid D\right)$ when relevant), so $\left(H \times \operatorname{id}_{\mathcal{J}}\right)^{*} J_{\text {taut }}$ can be considered an $\left(\omega_{\tilde{F}}, \tilde{T}\right)$-compatible complex structure on $\tilde{E} \mid\left(\left[0, \frac{1}{2}\right] \times X_{0} \times \mathcal{J}\right)$. Now fix some $J \in \mathcal{J}$ and pull it back to $\tilde{E} \mid\left(\left[\frac{1}{2}, 1\right] \times\right.$ $\left.X_{0} \times \mathcal{J}\right)$ by the same method, using the function $h^{\prime}(s, t)=h(1-s, t)$. Then the two complex structures agree over $\left\{\frac{1}{2}\right\} \times\left[0, \frac{1}{2}\right) \times \partial C \times \mathcal{J}$ (where they are both pulled back from $J_{C} \mid \partial C$ ), so together they define a complex structure $J_{\tilde{C}}$ on a neighborhood $\tilde{U}$ of $\tilde{C}$ as required. Construct the required local complex structures elsewhere on $\tilde{X}$ by (for example) pulling back the given structures on $X_{0}$ by $\pi_{X_{0}}$. The hypotheses of the lemma are now satisfied on $\tilde{X}$ if $D$ equals $C$ or $X$. For the general case, extend $J_{\tilde{D}}$ over a neighborhood $\tilde{V}$ of $\tilde{D}$ by a procedure similar to the one above, pulling back each local structure on $\tilde{E}$ (including $J_{\tilde{C}}$ ) along the product lines of the given collar of $\partial D$. The lemma gives a section $\tilde{J}: \tilde{X}=I \times X_{0} \times \mathcal{J} \rightarrow \operatorname{End}(\tilde{E})$, and hence a continuous map $\varphi: I \times \mathcal{J} \rightarrow \mathcal{C}(\xi)$ with image in $\mathcal{J}$. Since $\varphi \mid\{0\} \times \mathcal{J}=\operatorname{id}_{\mathcal{J}}$ and $\varphi \mid\{1\} \times \mathcal{J}=J$ (as a constant map into $\mathcal{J}$ ), $\varphi: I \times \mathcal{J} \rightarrow \mathcal{J}$ is the required contraction.

If $X_{0}$ is only locally compact, we wish to show that every map $S^{m} \rightarrow \mathcal{J}$ is homotopic to a constant map. But such a map can be interpreted as a section of the bundle $\pi_{X_{0}}^{*} \xi \rightarrow X_{0} \times S^{m}$ that is in fact a complex structure on $\pi_{X_{0}}^{*}\left(E \mid X_{0}\right) \rightarrow X_{0} \times S^{m}$. The previous argument with $S^{m}$ in place of $\mathcal{J}$ provides the required nullhomotopy.

\section{References}

[ABKLR] B. Aebischer, M. Borer, M. Kälin, Ch. Leuenberger and H. Reimann, Symplectic Geometry, Progress in Math. 124, Birkhäuser, 1994.

[A1] D. Auroux, Symplectic maps to projective spaces and symplectic invariants, Proceedings, Gökova Geometry-Topology Conference 2000, Turk. J. Math. 25 (2001), 1-42.

[A2] D. Auroux, Estimated transversality in symplectic geometry and projective maps, Proc. International KIAS Conference (Seoul 2000), to appear. 
[AK] D. Auroux and L. Katzarkov, The degree doubling formula for braid monodromies and Lefschetz pencils, J. Sympl. Geom., to appear.

[D] S. Donaldson, Lefschetz fibrations in symplectic geometry. Doc. Math. J. DMV, Extra Volume ICMII (1998), 309-314.

[G1] R. Gompf, A new construction of symplectic manifolds. Ann. of Math. 142 (1995), 527-595.

[G2] R. Gompf, The topology of symplectic manifolds, Proceedings, Gökova Geometry-Topology Conference 2000, Turk. J. Math. 25 (2001), 43-59.

[G3] R. Gompf, Symplectic structures from Lefschetz pencils in high dimensions, Proceedings of Cassonfest, Geometry and Topology, to appear.

[G4] R. Gompf, Locally holomorphic maps yield symplectic structures, is to appear in Comm. Anal. Geom.

[GS] R. Gompf and A. Stipsicz, 4-manifolds and Kirby calculus. Grad. Studies in Math. 20, Amer. Math. Soc., Providence, (1999).

[GH] P. Griffiths and J. Harris, Principles of algebraic geometry. Wiley, New York, (1978).

[Gr] M. Gromov, Partial Differential Relations, Ergebnisse der Mathematik, Series 3, Vol.9, Springer, Berlin, 1986.

[K] D. Kotschick, The Seiberg-Witten invariants of symplectic four-manifolds (after C.H. Taubes), Seminare Bourbaki 48ème année (1995-6), no. 812, Astérisque 241 (1997), 195-220.

[L] K. Lamotke, The topology of complex projective varieties after S. Lefschetz, Topology 20 (1981), 15-51.

[McS] D. McDuff and D. Salamon, Introduction to Symplectic Topology. Oxford University Press (1995).

[MS] J. Milnor and J. Stasheff, Characteristic Classes, Ann. of Math. Studies 76, Princeton Univ. Press, 1974.

[M] J. Moser, On the volume elements on a manifold. Trans. Amer. Math. Soc. 120 (1965), 286-294.

[Mu J. Munkres, Topology, Second Edition, Prentice Hall, 2000.

[P] R. Palais, Homotopy theory of infinite dimensional manifolds, Topology 5 (1966), 1-16.

[Ta] C. Taubes, The Seiberg-Witten invariants and symplectic forms. Math. Res. Letters 1 (1994), 809-822.

[T] W. Thurston, Some simple examples of symplectic manifolds. Proc. Amer. Math. Soc. 55 (1976), 467-468.

Department of Mathematics, The University of Texas at Austin, 1 University Station C1200, Austin, TX 78712-0257

E-mail address: gompf@math.utexas.edu

Partially supported by NSF grants DMS-9802533 and DMS-0102922. 